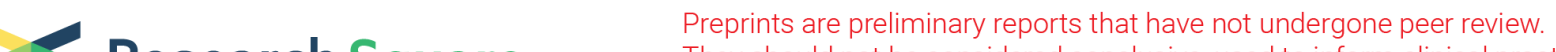 Research Square They should not be considered conclusive, used to inform clinical practice, or referenced by the media as validated information.
}

\section{Cisplatin-Resistant Gastric Cancer Cells Promote the Chemoresistance of Cisplatin-Sensitive Cells via Exosomal RPS3 Mediated PI3K-Akt-cofilin-1 Signaling Axis}

\section{Mengyao Sun}

Shanghai University of Traditional Chinese Medicine

\section{Bo Xu}

Shanghai University of Traditional Chinese Medicine

\section{Qiuxue Wu}

Shanghai University of Traditional Chinese Medicine

\section{Si Cai}

Shanghai University of Traditional Chinese Medicine

\section{Wenlian Chen}

Shanghai University of Traditional Chinese Medicine

\section{Dong Li}

Tongji University School of Medicine

\section{Hui Zhang}

Shanghai University of Traditional Chinese Medicine

\section{Qingfeng Tang ( $\nabla$ tangqingfeng126@126.com )}

\section{Research}

Keywords: gastric cancer, cisplatin resistance, exosome, RPS3, PI3K-Akt-cofilin-1

Posted Date: July 21st, 2020

DOI: https://doi.org/10.21203/rs.3.rs-44042/v1

License: (c) (i) This work is licensed under a Creative Commons Attribution 4.0 International License. Read Full License

Version of Record: A version of this preprint was published at Frontiers in Cell and Developmental Biology on February 11th, 2021. See the published version at https://doi.org/10.3389/fcell.2021.618899. 


\section{Abstract}

Background: Cisplatin is an important agent in first-line chemotherapy against gastric cancer (GC). However, consequential drug resistance limits its effectiveness for the treatment of GC. Exosomes which are loaded with proteins, lipids and RNAs, have been proven to transfer malignant phenotype. This study aims to explore the role and mechanism of exosomal RPS3 protein in transmitting a chemoresistance phenotype from cisplatin resistant to cisplatin sensitive gastric cancer cells.

Methods: A cisplatin resistant gastric cancer cell line SGC7901R was established by continuously grafting SGC7901S cells into cisplatin-containing culture medium. Exosomes from SGC7901R and SGC7901S were obtained and confirmed through ultracentrifuge and Nano Analyzer. By LC-MS/MS analysis methods, we detected the differentially expressed proteins in SGC7901R cells exosomes and SGC7901S cells exosomes. Western blotting was used to verify the differential expression of exosomal RPS3 between cisplatin resistant and parental cell lines. Subsequently, a series of in vitro assays and a xenograft tumor model were used to observe the functions of exosomal RPS3 protein in GC.

Results: SGC7901R cell derived exosomes were readily taken up by cisplatin sensitive SGC7901S cells, thus triggering off a phenotype of chemoresistance in the receptor cells. Subsequently, it was demonstrated that exosomal RPS3 was essential for inducing chemoresistance of receptor cells as shown by the acquisition of this phenotype in SGC7901S cells with enforced expression of RPS3. Further mechanism study demonstrated that cisplatin-resistant gastric cancer cells-derived exosomal RPS3 enhanced the chemoresistance of cisplatin-sensitive gastric cancer cells through the PI3K-Akt-cofilin-1 signaling pathway.

Conclusion: Cisplatin resistant gastric cancer cells communicate with sensitive cells through the intercellular delivery of exosomal RPS3 and activation of the PI3K-Akt-cofilin-1 signaling pathway. Targeting exosomal RPS3 protein in cisplatin resistant gastric cancer cells may thus be a promising strategy to overcome cisplatin resistance in gastric cancer.

\section{Background}

Gastric cancer ranks as the second most common malignant disease and the third leading cause of cancerassociated mortality in developing countries [1]. Chemotherapy is one of the principal therapeutic approaches used for the treatment of gastric cancer. Chemoresistance has been identified as a major problem in the process of cancer treatment. Cisplatin is widely used as a frontline chemotherapeutic agent for gastric cancer; however, chemoresistance limits the effectiveness of chemotherapy and results in treatment failure in the majority of cases [2,3]. In the past few years, some reports have partly shown the underlying mechanisms of cisplatin resistance in gastric cancer. However, the molecular mechanisms underlying cisplatin resistance remain to be further investigated.

Exosomes are a subset of extracellular microvesicles secreted from different types of cells [4]. More and more reports have indicated that exosomes play important roles in tumor growth, metastasis, 
angiogenesis, immune regulation by acting as information communicators between different types of cells [57]. Importantly, exosomes have recently been discovered anticipating in the regulation of chemoresistance in cancers [810]. As is known to all, exosomes are nanosized vesicles (about $100 \mathrm{~nm}$ ) loading a variety of kinds of cargo, including DNA, mRNA, miRNA, circRNA, proteins, etc [11]. Some reports have demonstrated that chemotherapy can change the exosomal composition secreted from tumor cells [1214], however, which exosomal cargos play key roles in regulating chemoresistance are not well understood.

In this study, a cisplatinresistant gastric cancer cell line SGC7901 (SGC7901R) was established, and the exosomal protein expression levels in SGC7901R cells and sensitive (SGC7901S) cells were investigated by LC-MS/MS and compared. RPS3 (Ribosomal Protein S3) was one of the highly expressed proteins in the exosome of SGC7901R cells in comparison with SGC7901S cell, which sparked our interest to explore the role and mechanism of exosomal RPS3 protein in transmitting a chemoresistance phenotype from cisplatinresistant to cisplatinsensitive gastric cancer cells.

\section{Materials And Methods}

Establishment of a cisplatinresistant SGC7901 cell line. The human gastric cancer cell line SGC7901 was obtained from the Cell Bank of Type Culture Collection of the Chinese Academy of Sciences (Shanghai, China). The cells were cultured in RPMI1640 medium (Gibco, Thermo Fisher Scientific, Inc.)

supplemented with $10 \%$ fetal bovine serum (FBS, Gibco, Thermo Fisher Scientifics, Inc.) and incubated at $37^{\circ} \mathrm{C}$ in a humidified incubator with $5 \% \mathrm{CO}_{2}$. Cisplatinresistant SGC7901R cells were established by continuous exposure to stepwiseincreasing concentrations of cisplatin (SigmaAldrich, Merck KGaA, Darmstadt, Germany). SGC7901 cells were initially cultured in RPMI1640 containing a low concentration

of cisplatin $(1 \mu \mathrm{g} / \mathrm{L})$. The cells were subsequently subcultured every 2 weeks in RPMI1640 with increasing concentrations of the drug, a $25 \%$ increase each time. Finally, cells that were viable in the cell culture medium with a high concentration of cisplatin $(1000 \mu \mathrm{g} / \mathrm{L})$ were designated as cisplatinresistant SGC7901R cells. These cells were maintained in the cisplatincontaining medium following induction but were cultured in drugfree medium for 1 week at $37^{\circ} \mathrm{C}$ prior to subsequent experimentation. Parental cells, denoted as SGC7901S, were cultured under the same conditions without treatment.

Exosome isolation and characterization. Gastric cancer cells were cultured in freshly prepared RPMI1640 medium containing exosomefree FBS for $48 \mathrm{~h}$ until cells had reached $90 \%$ confluence. Cell culture supernatants were collected and filtered using $0.22 \mu \mathrm{m}$ pore filters (Merck KGaA), followed by differential centrifugation at $4^{\circ} \mathrm{C}$ : $300 \mathrm{xg}$ for $10 \mathrm{~min}$ to remove cells, $2,000 \mathrm{xg}$ for $15 \mathrm{~min}$ to remove cell debris and $10,000 \times \mathrm{g}$ for $30 \mathrm{~min}$ to remove large particles. Then, the pellets containing exosomes were collected by spinning at $100,000 \times g$ for 70 min. After washing with PBS, the pellets were deal with ultracentrifugation (Beckman 70Ti rotor). The morphology of exosomes was examined via transmission electron microscopy. The number and size distribution of exosomes were detected by a LM10 nanoparticle characterization system (NanoSight, Malvern Instruments). The exosomal protein concentration was 
measured by the BCA method and exosomeassociated protein markers of HSP70, CD63, and CD9 expression were analyzed by Western blotting.

Exosome labeling and treatment. $8 \times 10^{4}$ of SGC7901S cells were seeded in 12 well plates and incubated at $37^{\circ} \mathrm{C}$ with red fluorescent dye CM-Dil (Invitrogen; Thermo Fisher Scientific, Inc.) at $37^{\circ} \mathrm{C}$ for $1 \mathrm{~h}$, washed with PBS and centrifuged at $110,000 \times \mathrm{g}$ at $4^{\circ} \mathrm{C}$ for 70 min to remove excess dye. Exosomes from SGC7901R cells were prelabeled with the green fluorescent dye PKH-67 (Invitrogen; Thermo Fisher Scientific, Inc.) at $37^{\circ} \mathrm{C}$ for $1 \mathrm{~h}$, washed with PBS and centrifuged at $110,000 \times \mathrm{g}$ at $4^{\circ} \mathrm{C}$ for $70 \mathrm{~min}$ to remove excess dye. Unlabeled exosomes were used as a negative control. The CM-Dil-labeled SGC7901S cells were incubated with PKH-67-labeled exosomes or unlabeled control exosomes for $4 \mathrm{~h}$. Then, SGC7901S cells were fixed with $4 \%$ paraformaldehyde at room temperature for $1 \mathrm{~h}$. Nuclear staining was performed with DAPI (40, 6-diamidino-2-phenylindole) at room temperature for $10 \mathrm{~min}$. Incorporation of exosomes into targeted SGC7901S cells was visualized by fluorescence microscopy (Zeiss AG, Germany).

Cell proliferation analysis. SGC7901R and SGC7901S cells were seeded in 96 well plates (5,000 cells/well) and exposed to increasing concentrations of cisplatin for $48 \mathrm{~h}$ at $37^{\circ} \mathrm{C}$. The concentrations of cisplatin used for the drug doseresponse curve analysis of SGC7901R and SGC7901S cells were $0,125,250,500$, $1,000,2,000,4,000,8,000$, and $16,000 \mu \mathrm{g} / \mathrm{L}$. The proliferative ability of cells was determined with a Cell Counting Kit8 (CCK8) kit (MedChemExpress, Monmouth Junction, USA) according to the manufacturer's protocols and presented as cell viability (\%). The absorbance was measured at $450 \mathrm{~nm}$ using ausing a Varioskan Flash Microplate Reader (Thermo Fisher Scientific, Waltham, MA, USA). The halfmaximal inhibitory concentration (IC50) of drugs was calculated using GraphPad Prism version 8.0 software (GraphPad Software, Inc., La Jolla, CA, USA). Exosomes were isolated from SGC7901S cells, SGC7901R cells, and cancer cells following transfection with oligonucleotides (described below). For exosome treatment analysis, each well in a 96well plate was seeded with 1,000 cells and loaded with exosomes at $100 \mu \mathrm{g} / \mathrm{mL}$ for $48 \mathrm{~h}$ for functional analysis at $37^{\circ} \mathrm{C}$, and the untreated cells served as the control. The cell culture medium was then removed and fresh medium containing the IC50 concentration of cisplatin was added to each well for $48 \mathrm{~h}$. At the end of treatment, cell proliferation was measured.

LC-MS/MS analysis. $20 \mu g$ proteins from each sample were denatured using $8 \mathrm{M}$ urea, reduced with $10 \mathrm{mM}$ dithiothreitol (DTT), and alkylated using $100 \mathrm{mM}$ iodoacetamide. The samples were then proteolytically digested with endoproteinase LysC overnight at room temperature, following by digestion with trypsin for $15 \mathrm{~h}$ at $37^{\circ} \mathrm{C}$. The resulting peptide mixtures were extracted using a peptide extract solution ( $50 \% \mathrm{ACN}, 0.1 \% \mathrm{TFA}$ ) for $30 \mathrm{~min}$ at $37^{\circ} \mathrm{C}$. Then the samples were dried and solubilized in the sample loading buffer containing $0.1 \%$ formic acid. Each sample of about 3-5 $\mu \mathrm{g}$ was analyzed by reversed-phase nano-liquid chromatography-tandem mass spectrometry (LC-MS/MS) (Thermo Scientific). The source data from three technical replicates of each sample were analyzed by searching the Uniprot human database with MaxQuant and Perseus software. Lable free quantitative (LFQ) values represent protein abundance. The False discovery rate (FDR) values at the protein and peptide levels were set to $1 \%$. Only those proteins quantified in at least two out of three replicates in at least one group remain for 
further analysis. The multiple sample ANOVA test was executed and corrected for multiple hypotheses testing using a cutoff of FDR $<0.05$.

RTqPCR. Total RNA from cells was extracted using TRIzol (Invitrogen; Thermo Fisher Scientific, Inc.). RNA concentrations were measured with a NanoDrop ND-1000 instrument (NanoDrop Technologies; Thermo Fisher Scientific, Inc.). cDNA was produced by reverse transcription using the SuperScript ${ }^{\circledR}$ III RT-PCR kit according to the manufacturer's instructions (Thermo Fisher Scientific, Inc.). The amplification of fluorescence signals was detected by a fluorescence thermal cycler (BioRad Laboratories, Inc., USA). RTqPCR was performed using the following primers: RPS3 sense, 5'-GCGAGTTACACCAACCAGGA-3' and antisense, 5'- ATGAACCGCAGCACACCATA-3'; $\beta$-actin sense, 5'-AGCAGCATCGCCCCAAAGTT-3' and antisense, 5'-GGGCACGAAGGCTCATCATT-3'. Bactin was set as an internal control for cellular mRNAs. The parameters for PCR quantification were as follows: 2 min at $95^{\circ} \mathrm{C}$, followed by 40 cycles of 15 secs at $95^{\circ} \mathrm{C}$ and 30 secs at $60^{\circ} \mathrm{C}$. The results of qPCR were defined using the quantification cycle $(\mathrm{Cq})$, and $2^{-\triangle \Delta \mathrm{Cq}}$ was used to calculate the relative expression levels (15).

Western blotting. Cellular proteins were extracted with RIPA lysis buffer (Beyotime Institute of Biotechnology). Protein concentration was determined by the BCA method. A total of $40 \mu \mathrm{g}$ of protein for each group was separated on $12 \%$ SDSPAGE gels and transferred to $0.45 \mu \mathrm{m}$ PVDF membranes (EMD Millipore). Membranes were then blocked with $5 \%$ BSA and incubated with primary antibodies at $4{ }^{\circ} \mathrm{C}$ overnight against HPS70 (4876, 1: 1,000), CD9 (ab92726, 1: 1,000), CD63 (ab193349, 1: 1,000), RBP4(ab233138, Abcam), RPSA(ab133645, Abcam), RPS3(9538, CST), RPS20 (ab133776, Abcam), RPS14 (ab246916, Abcam), RPL4 (ab234829, Abcam), RPL13 (ab134961, Abcam), HSPD1 (ab46798, Abcam), HSPA8 (8444, CST), P-gp (13342, CST), p-cofilin-1 (3313, CST), cofilin-1 (5175, CST), PP1 (sc7482, Santa Cruz), PP2A (9780, CST). Anti- $\beta$-actin rabbit monoclonal antibody (1:5,000, ab179467, Abcam) was used as the internal control. The membranes were then washed 3 times and incubated at $37^{\circ} \mathrm{C}$ for $1 \mathrm{~h}$ with an HRP-conjugated anti-rabbit secondary antibody (1:5,000, ab97051, Abcam). Bands were visualized by chemiluminescence using immobilon ECL Ultra Western HRP Substrate (Merck KGaA) according to the manufacturer's protocols.

Plasmid construction and cell transfection. The recombinant plasmids containing the pcDNA3.1 empty vector, pcDNA3.1-RPS3, pLV4-shRNA/RPS3, and the non-targeting control pLV4-shRNA-negative control (NC) were produced by GeneChem, Inc (Shanghai Genechem Company Ltd., China). All the transfections were performed using Lipofectamine 3000 reagent (Invitrogen; Thermo Fisher Scientific, Inc.) in accordance with the manufacturer's instructions. To improve the transfection efficiency in neuronal cells, the positively transfected cells were subsequently screened by flow cytometry following transfection with a GFP-labeled for $72 \mathrm{~h}$. The transfection efficiency was evaluated by fluorescence microscopy observation.

ICP-MS Analysis. SGC-7901R or SGC-7901S cells were washed with PBS for three times, and then the cells were digested and collected. $500 \mu \mathrm{L} \mathrm{ddH_{2 }} \mathrm{O}$ was added and the cells were broken by freezing and thawing with liquid nitrogen repeatedly followed by sonication. The samples were centrifuged at 
$14,000 \mathrm{rpm} / \mathrm{min}$ for $10 \mathrm{~min}$, collected the supernatant, dried under vacuum for $24 \mathrm{~h}$, and dissolved in PBS. The dissolved samples were digested by $2 \mathrm{~mL}$ nitric acid followed by $1 \mathrm{~mL}$ perchloric acid, cooled down, set to a constant volume of $10 \mathrm{~mL}$, and ultrafiltrated. The ultrafiltrated samples $(10 \mathrm{~mL})$ were diluted using a Gilson ASPEC XLi programme to deliver $1.8 \mathrm{~mL}$ of iridium internal standard $(0.005 \mathrm{mg} / \mathrm{mL}$, in $1 \%$ nitric acid) and mixed thoroughly. Intracellular accumulation of DDP in each cell sample was determined by ultrasensitive multi-collector inductively coupled with mass spectrometry (ICP-MS) as previously described [16].

Flow Cytometry Analysis. SGC-7901R or SGC-7901S cells were plated in 6-well plates at $2 \times 10^{5}$ cells/well in RPMI 1640 medium with 10\% FBS. The cells were collected, washed with cold PBS twice, and resuspended gently in $400 \mu \mathrm{L}$ binding buffer. $5 \mu \mathrm{L}$ AnnexinV FITC was added to the above cells solution, gently vortexed, and incubated for $10 \mathrm{~min}$ at $4{ }^{\circ} \mathrm{C}$ avoiding the light. $10 \mu \mathrm{L}$ propidium iodide (PI) was added and cultured for another 5 min. Flow cytometry was then conducted using FACSCalibur Flow Cytometry (BD Biosciences, USA), and the results were analyzed with CellQuest software.

Tumor Mouse Model. SGC-7901S cells were harvested in serum-free PBS and $100 \mu \mathrm{L}$ single-cell suspensions $\left(2 \times 10^{7}\right.$ cells $\left./ \mathrm{mL}\right)$ were injected into the subcutaneous area of male BALB/c nude mice (4-6 weeks old, SLAC Laboratory Lab, Shanghai, China). When the tumors reached an average size of $100 \mathrm{~mm}^{3}$, the mice were randomized into 5 groups $(n=8)$. Mice in group 1 were administered with normal saline daily, mice in group 2 were administered with $10 \mu \mathrm{g} / \mathrm{mL}$ DDP, mice in groups 3,4 , and 5 proceeded intragastric administration of control exosomes, shRNA/RPS3-exosomes, over/RPS3-exosomes, plus $10 \mu \mathrm{g} / \mathrm{mL}$ DDP, respectively. The length and width of tumors were recorded every 7 days. After 35 days, animals were sacrificed by cervical dislocation in deep anesthesia of $\mathrm{CO}_{2}$, and primary tumors were surgically removed and weighed. Tumor sizes were evaluated using the formula: length $\times$ width ${ }^{2 \times 0} .52$. The primary tumors were analyzed by TUNEL assay, and p-PI3K, p-AKT, PP1, and PP2A proteins were detected by immunohistochemistry.

TUNEL assay. TUNEL assay (a DeadEndTM Colorimetric TUNEL System kit, Promega Corp.) was performed to detect the apoptosis of the subcutaneous tumors in accordance with the manufacturer's instructions. The observation and capture of digital images were conducted using a Nikon E80i microscope (Nikon Corp.).

Immunohistochemistry (IHC). Paraffin-embedded tumor tissue samples were selected for IHC. IHC for $\mathrm{p}$ PI3K (17366, CST), p-AKT (4060, CST), PP1 (sc-7482, Santa Cruz) and PP2A (9780, CST) was carried out using a rabbit monoclonal or polyclonal primary antibody overnight at $4{ }^{\circ} \mathrm{C}$, an HRP-conjugated goat antirabbit IgG secondary antibody $\left(1: 1,000\right.$, ab150077, Abcam) for $1 \mathrm{~h}$ at $37^{\circ} \mathrm{C}$, and $\mathrm{DAB}$ staining solution (ab64238, Abcam) for $10 \mathrm{~min}$ at room temperature. The staining results were assessed with a Nikon E80i microscope (Nikon Corp.).

Tissue immunofluorescence. Dissected tissues were fixed in a mixture of $2 \%$ PFA and $20 \%$ sucrose solution for $24 \mathrm{~h}$ at room temperature and then embedded in Tissue-Tek O.C.T. (Electron Microscopy 
Sciences). Blocks were frozen in a dry ice and ethanol bath. For immunofluorescence, $6 \mu \mathrm{m}$ O.C.T. tissue cryosections were stained with antibodies against P-cofilin-1(3313, CST). Secondary antibodies conjugated to Alexa Fluor 594 (A-11032, 1: 1,000) were used (Life Technologies). Nuclear staining was done with DAPI (40, 6-diamidino-2-phenylindole). Immunofluorescence images were taken with fluorescence microscopy (Zeiss AG, Germany). All experiments were conducted according to instructions from the antibody manufacturer.

Statistical analysis. All data are presented as the mean \pm standard error of the mean from at least three independent experiments. A Student's ttest, oneway analysis of variance, and a Tukey's post hoc test were performed using GraphPad Prism 8.0 software. $P<0.05$ was considered as statistically significant difference.

\section{Results}

SGC7901Rexosomes induce a cisplatinresistant phenotype in SGC7901S cells. Several studies have demonstrated that chemo-resistant tumor cells can release exosomes to chemo-sensitive tumor cells and transmit drug resistance during tumorigenesis (1214). To analyze whether SGC7901R cellderived exosomes may confer a malignant phenotype on cisplatinsensitive tumor cells, exosomes from the culture medium of SGC7901R and SGC7901S cells were isolated. Purified exosomes from the cultures exhibited typical cupshaped morphology by transmission electron microscopy analysis (Fig. 1A). Using the LM10 nanoparticle characterization system, we detected the size and particle number of the purified exosomes. The mean size of SGC7901Rexosomes and SGC7901Sexosomes were both between $100 \mathrm{~nm}$ and $200 \mathrm{~nm}$ (Fig. 1B), which was corresponded with the reported size of exosomes (29). Moreover, the particle numbers for two tumor cellderived exosomes were both more than $2.0 \times 10^{7}$ particles $/ \mathrm{ml}$ (Fig. 1B). No significant difference in exosome quantities extracted from SGC7901R and SGC7901S cells was discovered. Next, the exosome markers were detected by Western blotting. Both of the two tumor cellderived exosomes showed positive expression of exosome markers, including HSP70, CD63, and CD9 (Fig. 1C).

To demonstrate that exosomes could be taken up by the recipient cells, CMDil-labeled SGC7901S cells (Red) were incubated with PKH67labeled exosomes (green) derived from SGC7901R cells. In Fig. 1D, we showed that PKH67 green fluorescence signals were visible around the nuclei and were also in the cytoplasm of SGC7901S cells following exposure to SGC7901Rderived exosomes. However, the negative control did not exhibit any green fluorescence (data not shown). This suggested the effective uptake of PKH67labeled exosomes by SGC7901S cells. Subsequently, to investigate whether SGC7901Rexosomes were responsible for the spread of chemoresistance, SGC7901S cells were incubated with SGC7901Rexosomes for $48 \mathrm{~h}$, and then both the control SGC7901S cells and SGC7901S cells pre-treated with SGC7901Rexosomes were treated with cisplatin for chemosensitivity analysis. CCK8 assays revealed that SGC7901Rexosome treatment significantly reduced the chemosensitivity of SGC7901S cells, while SGC7901Sexosomes had no such effect compared with the control (Fig. 1E). These results 
demonstrated that the cisplatinresistant phenotype in SGC7901S cells could be induced by SGC7901Rexosomes.

Cisplatinresistant gastric cancer cell line SGC7901R exhibits upregulated levels of RPS3 protein. Since SGC7901Rexosomes can induce the cisplatinresistant phenotype of SGC7901S cells, it was very necessary to compare the contents in the exosomes of SGC7901R and SGC7901S cells. Although the exosomes contain a variety of kinds of cargo, including DNA, mRNA, miRNA, circRNA, proteins, etc., here in our study we just consider the protein contents that might play key roles in cisplatinresistance delivery. By LC-MS/MS analysis methods (Fig. 2A), we detected the differentially expressed proteins in SGC7901R cells exosomes and SGC7901S cells exosomes. The cluster analysis in Fig. 2B demonstrated that many differentially expressed proteins were discovered between SGC7901R cells exosomes and SGC7901S cells exosomes. By GO function and KEGG pathway analysis, many important proteins were screened out, including RBP4, RPSA, RPS3, RPS20, RPS14, RPL4, RPL13, HSPD1, HSPA8, etc. (Fig. S1, Fig. S2, Fig. S3). Next, Western blotting was performed to validate the significant differentially expressed proteins in the exosomes of SGC7901R and SGC7901S cells (Fig. 2C). The results showed that several differentially expressed proteins detected by LC-MS/MS also showed significant difference by immunoblotting validation, including RBP4, RPSA, RPS3, RPS20, and RPS14 (Fig. 2D), while other proteins showed little difference (Fig. 2E). Considering the difference was the most significant and acting as an important joint of the PPI network, as well as the preliminary experiments (data not shown), RPS3 was selected as the targets for further research.

Exosomal transfer of RPS3 promotes the cisplatin chemoresistance in SGC7901S cells. To further validate that exosomal RPS3 is involved in the induction of a cisplatinresistant phenotype, RPS3 was overexpressed or knockdown in SGC7901R cells. RTqPCR and Western blotting analysis demonstrated that RPS3 was successfully overexpressed or knockdown in RPS3-overexpressing or -silencing SGC7901R cells, compared with their respective controls (Fig. 3A, 3B). Then, the exosomes derived from RPS3-overexpressing or -silencing SGC7901R cells were isolated, accompanying with their respective controls. Western blotting results showed that RPS3 protein was enriched in exosomes derived from RPS3-overexpressing SGC7901R cells, but down-regulated in exosomes derived from RPS3-silencing SGC7901R cells (Fig. 3C, 3D).

Next, to evaluate whether the exosomal transfer of RPS3 promotes the cisplatin chemoresistance in SGC7901S cells, SGC7901S cells were pre-treated with exosomes isolated from RPS3-overexpressing or silencing SGC7901R cells, in compared with their respective controls, and then different concentrations of cisplatin were added to above cells. The results revealed that the proliferation of SGC7901S cells was significantly reduced by RPS3-silencing SGC7901R cellsexosomes treatment but increased by RPS3overexpressing SGC7901R cellsexosomes treatment following incubation with cisplatin compared with the control exosomes (Fig. 3E). Then, using ICP-MS for determination of the changed intracellular DDP concentration in SGC-7901S cells, we found that, when $6.73 \mu \mathrm{g} / \mathrm{mL}$ DDP exposed to above cells for 48 hours, the intracellular accumulation of DDP increased in SGC7901S cells pre-treated with RPS3-silencing SGC7901R cellsexosomes, but reduced in SGC7901S cells pre-treated with RPS3-overexpressing 
SGC7901R cellsexosomes compared with the control exosomes (Fig. 3F). Subsequently, we detected the protein expression of RPS3 and P-gp in SGC7901S cells pre-treated with exosomes derived from RPS3overexpressing or -silencing SGC7901R cells. The results showed that RPS3 and P-gp were both reduced in SGC7901S cells pre-treated with RPS3-silencing SGC7901R cellsexosomes, but increased in SGC7901S cells pre-treated with RPS3-overexpressing SGC7901R cellsexosomes compared with the control exosomes (Fig. 3G, 3H). These results indicated that exosomal transfer of RPS3 protein may be involved in the induction of a cisplatinresistant phenotype.

\section{RPS3-riched exosomes from SGC7901R cells decreased the apoptosis of SGC7901S cells induced by} DDP via affecting the mitochondrial translocation of cofilin-1. Flow cytometry was performed to see the effect of exosomes derived from RPS3-overexpressing or -silencing SGC7901R cells on the cell cycle and apoptosis of SGC-7901S cells following incubation with cisplatin. The results showed that pretreatment of exosomes derived from RPS3-silencing SGC7901R cells led to the reduction of SGC-7901S cells in G2/M phages compared with the control exosomes, while the exosomes derived from RPS3overexpressing SGC7901R cells did the opposite effect (Fig. 4A, 4B). In addition, pretreatment of exosomes derived from RPS3-silencing SGC7901R cells increased DDP-induced cell apoptosis of SGC$7901 \mathrm{~S}$ cells compared with the control exosomes, while the exosomes derived from RPS3-overexpressing SGC7901R cells did the opposite effect (Fig. 4C, 4D). Furthermore, by Western blotting analysis, we showed that RPS3-riched exosomes from RPS3-overexpressing SGC7901R cells reduced the mitochondrial translocation of cofilin-1 in SGC-7901S cells compared with the control exosomes, while RPS3-reduced exosomes from RPS3-silencing SGC7901R cells did the opposite effect (Fig. 4E, 4F, 4G, $4 \mathrm{H})$. These data implied that RPS3-riched exosomes from SGC7901R cells decreased the apoptosis of SGC7901S cells induced by DDP via affecting the mitochondrial translocation of cofilin-1.

\section{Overexpression of RPS3 directly in SGC7901S cells exhibits similar effects to treatment with}

SGC7901Rexosomes. Our above data suggested that RPS3 expression levels were significantly elevated in SGC7901R cells, and SGC7901Rexosome treatment was proposed to confer cisplatinresistant phenotypes to SGC7901S cells. To further investigate the role of RPS3 in the regulation of cisplatin chemoresistance, RPS3 was directly overexpressed or knockdown in SGC7901S cells. RTqPCR and Western blotting analysis demonstrated that RPS3 was successfully overexpressed or knockdown in RPS3-overexpressing or -silencing SGC7901S cells, compared with their respective controls (Fig. 5A, 5B). Next, the CCK-8 assay revealed that the proliferation of SGC7901S cells was significantly reduced by RPS3-silencing but increased by RPS3-overexpressing following incubation with cisplatin compared with the controls (Fig. 5C). Then, the ICP-MS analysis demonstrated that, when $6.73 \mu \mathrm{g} / \mathrm{mL}$ DDP was exposed to the above cells for 48 hours, the intracellular accumulation of DDP increased in RPS3-silencing SGC7901S cells but reduced in RPS3-overexpressing SGC7901S cells compared with their controls (Fig. 5D). Subsequently, we detected the protein expression of P-gp in RPS3-overexpressing or -silencing SGC7901R cells, the results showed that P-gp was reduced in RPS3-silencing SGC7901S cells, but increased in RPS3-overexpressing SGC7901S cells compared with their controls (Fig. 5E, 5F). These results indicated that overexpression of RPS3 directly in SGC7901S cells also exhibits similar effects to treatment with SGC7901R exosomes. 
Next, flow cytometry was performed to see the effect of RPS3 overexpression and knockdown directly in SGC7901S cells on the cell cycle and apoptosis of SGC-7901S cells following incubation with cisplatin. The results showed that knockdown of RPS3 directly in SGC7901S cells led to the reduction of SGC7901S cells in G2 phages compared with the control, while the overexpression of RPS3 directly in SGC7901S cells did the opposite effect (Fig. 5G). In addition, knockdown of RPS3 directly in SGC7901S cells increased DDP-induced cell apoptosis of SGC-7901S cells compared with the control, while the overexpression of RPS3 directly in SGC7901S cells did the opposite effect (Fig. 5H). Furthermore, by Western blotting analysis, we showed that RPS3-overexpressing SGC7901S cells reduced the mitochondrial translocation of cofilin-1 in SGC-7901S cells compared with the control, while RPS3silencing SGC7901S cells did the opposite effect (Fig. 5J). Additionally, we also found that RPS3overexpressing SGC7901S cells reduced the expression of PP1 and PP2A in SGC-7901S cells compared with the control, while RPS3-silencing SGC7901S cells did the opposite effect (Fig. 5I, 5J). These data suggested that overexpression of RPS3 directly in SGC7901S cells decreased the apoptosis of SGC7901S cells induced by DDP via affecting the mitochondrial translocation of cofilin-1 and the expression of PP1 and PP2A, which were closely associated with PI3K-Akt signaling pathway.

\section{Impact of RPS3-riched exosomes from SGC7901R cells on the growth of subcutaneous xenograft of} SGC-7901S cells in nude mice. To explore whether RPS3-riched exosomes from SGC7901R cells enhanced the proapoptotic effect of DDP in vivo, we employed a subcutaneous xenograft of SGC7901 cells in nude mice model. As shown in Fig. 6A, pretreatment of exosomes derived from RPS3overexpressing SGC7901R cells reduced the inhibitory effect of DDP on the tumor growth in compared with DDP $(0.6 \mathrm{mg} / \mathrm{kg})$ alone, while the pretreatment of exosomes derived from RPS3-silencing SGC7901R cells did the opposite effect (Fig. 6A). The final tumor resection and weights calculation also showed that pretreatment of exosomes derived from RPS3-overexpressing SGC7901R cells following incubation with cisplatin showed the highest tumor weight compared with DDP alone, while the pretreatment of exosomes derived from RPS3-silencing SGC7901R cells following incubation with cisplatin did the opposite effect (Fig. 6B, 6C). Next, ICP-MS analysis was applied to investigate the DDP concentration in the subcutaneous xenograft of SGC7901S cells with indicated treatments. The results showed that pretreatment of exosomes derived from RPS3-overexpressing SGC7901R cells following incubation with cisplatin showed the highest levels of DDP concentration in the xenograft tumor compared with DDP alone, while the pretreatment of exosomes derived from RPS3-silencing SGC7901R cells following incubation with cisplatin did the opposite effect (Fig. 6D).

Then, a TUNEL assay was performed to observe the apoptosis of tumor cells in the subcutaneous xenograft of SGC7901S cells with indicated treatments. The results in Fig. 6E showed that pretreatment of exosomes derived from RPS3-overexpressing SGC7901R cells following incubation with cisplatin showed the least apoptotic tumor cells in the xenograft tumor in compared with DDP alone, while the pretreatment of exosomes derived from RPS3-silencing SGC7901R cells following incubation with cisplatin did the opposite effect. Additionally, we detected the expression of p-PI3K, p-AKT, PP1, and PP2A in the xenograft tumor tissues by immunohistochemistry $(\mathrm{IHC})$. The IHC images demonstrated that pretreatment of exosomes derived from RPS3-overexpressing SGC7901R cells following incubation with 
cisplatin showed the highest expression levels of p-PI3K, p-AKT, PP1, and PP2A in the xenograft tumor in compared with DDP alone, while the pretreatment of exosomes derived from RPS3-silencing SGC7901R cells following incubation with cisplatin did the opposite effect (Fig. 6F). Finally, we detected the p-cofilin1 expression by immunofluorescence in the subcutaneous xenograft of SGC7901S cells with indicated treatments. The immunofluorescence pictures showed that pretreatment of exosomes derived from RPS3-overexpressing SGC7901R cells following incubation with cisplatin showed the highest expression levels of p-cofilin-1 in the xenograft tumor in compared with DDP alone, while the pretreatment of exosomes derived from RPS3-silencing SGC7901R cells following incubation with cisplatin did the opposite effect (Fig. 6G).

All the above data suggested that RPS3-riched exosomes from SGC7901R cells blocked the inhibitory effect of DDP on the growth of subcutaneous xenograft in nude mice by enhancing the cisplatin chemoresistance in SGC7901S cells.

\section{Discussion}

Cisplatin has been widely used in tumor therapy, but the development of chemoresistance is often observed in patients with tumors [2,3]. At present, cisplatin resistance in tumors has been reported to be associated with underlying mechanisms, including increased drug efflux, alteration of intercellular signaling, tubulin mutation, and overexpression of ßtubulin isotype composition[17, 18]. Recently, it has been demonstrated that drugresistant cancer cells could transmit chemoresistant phenotypes to chemosensitive cancer cells via exosomes [8]. Whether the acquisition of cisplatin resistance by gastric cancer cells occurs in this way remains unknown.

Increasing evidence suggests that tumor cellderived exosomes provide critical signals in the development of chemoresistance [810]. Several studies have revealed that the delivery of key molecules by exosomes to tumor cells could lead to chemoresistance $[19,20]$. Among the main components in exosomal cargo, some molecules have been discovered acting as the vital regulating effect on chemoresistance [21, 22]. Several studies have demonstrated that exosomal miRNAs, IncRNAs, mRNA, and proteins could be shuttled from chemoresistant to chemosensitive cancer cells to transmit chemoresistance. For example, exosomal miR2223p from the gemcitabineresistant lung cancer line A549 enhanced gemcitabine resistance in parental sensitive cells [23]. A549/DDPexosomes conferred resistance to DDP in recipient cells depending on exosomal miR1005p [24]. Qu et al. identify IncARSR as a mediator of sunitinib resistance in renal cell carcinoma by acting as a competing endogenous RNA for miR-34 and miR-449, thereby increasing expression of their targets AXL and c-MET [25]. In breast cancer, Xu et al. demonstrated that exosomal transfer of IncRNA urothelial cancer associated-1 (UCA1) can increase tamoxifen resistance in estrogen receptor-positive MCF-7 cells through the mTOR signaling pathway [26]. Ciravolo et al. found that exosomes expressing full length human epidermal growth factor 2 molecules enable them to bind and sequester Trastuzumab, thus lowering the therapeutic effectiveness of Trastuzumab in breast cancer [27]. Colon cancer cell exosomes highly enriched in Nterminal truncated isoforms of p73 mRNA were able to confer drug resistance on recipient cells [19]. Exosomes from 
glioblastoma cancer cells harboring a protein tyrosine phosphatase receptor type Z1MET fusion conferred temozolomide resistance on parental cells [20]. These findings demonstrated that exosomes could transfer intercellular drug resistance from drugresistant to drugsensitive cancer cells.

To improve understanding of the underlying mechanisms of chemoresistance, chemoresistant cancer cells may be an ideal cell model for investigation. The role of exosomes secreted from chemoresistant cancer cells in the induction of chemoresistance has been studied. To investigate the mechanism of cisplatin resistance in gastric cancer cells, the cisplatinresistant gastric cancer cell line SGC7901R was established in the present study. SGC7901R cellderived exosomes could be effectively taken up by cisplatinsensitive SGC7901S cells and the receptor cells exhibited remarkably chemoresistance phenotypes. Consistently, it was demonstrated that SGC7901R derivedexosomes conferred a cisplatinresistant phenotype in SGC7901S cells. Presently, a large number of differentially expressed molecules in the exosomes are discovered by RNA sequencing or proteomics, many of which are potentially associated with chemoresistance, however, quite a lot of them are little investigated.

In this study, cisplatinresistant gastric cancer cell line SGC7901R was determined by LC-MS/MS with increased exosomal levels of RPS3 protein. RPS3, a component of the 40S ribosomal small subunit, is mainly involved in ribosomal maturation and initiation of translation [28]. RPS3 has various extraribosomal functions, including cell signaling [29], transcriptional regulation [30], DNA repair [31], etc. In addition, both up-regulation and intrinsic dysfunctions in ribosomes result in an increased incidence of tumors, and RPS3 is involved in radioresistance or invasion of tumor cells[32, 33]. Huang et al. showed that Vitamin $D$ promotes the cisplatin sensitivity of oral squamous cell carcinoma by inhibiting LCN2modulated NF-KB pathway activation through RPS3 [34]. Zhao et al. unveiled a novel extra-ribosomal role of RPS3 in facilitating hepatocarcinogenesis via the post-transcriptional regulation of SIRT1 expression and proposes that the RPS3/SIRT1 pathway serves as a potential therapeutic target in HCC [35]. A previous study showed that RPS3 was secreted into the extracellular environment in a dimeric form. All the reported data suggested that RPS3 may be a putative marker for malignant tumors.

In the present study, our data demonstrated that RPS3 protein was enriched in SGC7901Rexosomes and could be delivered into SGC7901S cells. Overexpression of RPS3 directly in SGC7901S cells can also result in similar phenotypic effects as treatment with SGC7901R exosome, which then capable of inducing the malignant phenotype in the sensitive cells. Further mechanism study demonstrated that cisplatin-resistant gastric cancer cells-derived exosomal RPS3 could enhance the chemoresistance through the PI3K-Akt-cofilin-1 signaling pathway. In our previous studies, we have demonstrated that cofilin-1 signaling pathway is involved in the mitochondrial apoptosis of cisplatin-resistant gastric cancer cells [36]. The phosphoric acid lipases, PP1, PP2A, and SSH, can activate cofilin-1, allowing cofilin-1 to bind F-actin and promote the depolymerization of actin filaments. Previous studies demonstrated that changes in cofilin-1 or p-cofilin-1 patterns played an important role in multidrug resistance in tumor cells[37, 38]. All the findings demonstrated that cisplatinresistant gastric cancer cells communicate with sensitive cells through the delivery key exosomal protein RPS3 and the activation of PI3K-Akt-cofilin-1 signaling pathway. 
In conclusion, our present study demonstrated that RPS3 protein expression levels were significantly elevated in SGC7901R cells, which was selectively sorted into SGC7901R cellderived exosomes. Exosomal delivery of RPS3 protein may induce chemoresistance phenotypes from cisplatinresistant gastric cancer cells to sensitive cancer cells by regulating the PI3K-Akt-cofilin-1 signaling pathway. Therefore, exosomal RPS3 protein in cisplatinresistant gastric cancer cells may thus be a promising strategy to overcome cisplatin resistance in gastric cancer.

\section{Abbreviations}

GC: Gastric cancer; RPS3: Ribosomal Protein S3; DDP: Cisplatin; DTT: Dithiothreitol; HE: Hematoxylin and eosin; IHC: Immunohistochemistry; RT-qPCR: Real-time quantitative polymerase chain reaction; ICP-MS: ultrasensitive multi-collector inductively coupled with mass spectrometry; LC-MS/MS: reversed-phase nano-liquid chromatography-tandem mass spectrometry; CCK-8: Cell counting kit-8; PI: Propidium lodide .

\section{Declarations}

\section{Acknowledgements}

We thank Professor Ming Guan from Department of Clinical Laboratory and Central Laboratory, Huashan Hospital, Shanghai Medical School, Fudan University.

\section{Authors' contributions}

QT, $\mathrm{HZ}$ and DL were responsible for designing the experiments and research supervision. MS, BX and QW were responsible for conducting experiments, acquisition of data and analysis. DL helped to plot figures and provide critical comments. SC and WC carried out the statistical analysis. MS and QW wrote the manuscript. All authors reviewed and approved the final version.

\section{Funding}

This work was supported by the Natural Science Foundation of Shanghai (No.19ZR1447800); The Health System independent Innovation Science Foundation of Shanghai Putuo District (No. ptkwws201704); Shanghai Municipal Health Commission Project (No. 20194Y0213).

\section{Availability of data and materials}

All of the data and materials in this paper are available when requested.

\section{Ethics approval and consent to participate}

Not applicable.

\section{Consent for publication}


Not applicable.

\section{Competing interests}

The authors declare that they have no competing interests.

\section{References}

1. Bray F, Ferlay J, Soerjomataram I, Siegel RL, Torre LA, Jemal A. Global cancer statistics 2018: GLOBOCAN estimates of incidence and mortality worldwide for 36 cancers in 185 countries. CA Cancer J Clin. 2018;68(6):394-424.

2. Guo Z, Wang X, Lin R, Chen L, Fan N, Chen Y, et al. Cisplatin-based regimens as first-line treatment in advanced gastric cancer. J Chemother. 2015;27:94-98.

3. Zhang D, Fan D. Multidrug resistance in gastric cancer: Recent research advances and ongoing therapeutic challenges. Expert Rev Anticancer Ther. 2007;7(10):1369-1378.

4. Zhang J, Li S, Li L, Li M, Guo C, Yao J, et al. Exosome and exosomal microRNA: Trafficking, sorting, and function. Genomics Proteomics Bioinformatics. 2015;13(1):17-24.

5. Meehan K, Vella LJ. The contribution of tumour-derived exosomes to the hallmarks of cancer. Crit Rev Clin Lab Sci. 2016;53(2):121-131.

6. Ruivo CF, Adem B, Silva M, Melo SA. The biology of cancer exosomes: Insights and new perspectives. Cancer Res. 2017;77(23):6480-6488.

7. Brinton LT, Sloane HS, Kester M, Kelly KA. Formation and role of exosomes in cancer. Cell Mol Life Sci. 2015; 72(4):659-671.

8. Sharma A. Chemoresistance in cancer cells: Exosomes as potential regulators of therapeutic tumor heterogeneity. Nanomedicine. 2017;12(17):2137-2148.

9. Butera G, Pacchiana R, Donadelli M. Autocrine mechanisms of cancer chemoresistance. Semin Cell Dev Biol. 2018;78:3-12.

10. Sousa D, Lima RT, Vasconcelos MH. Intercellular transfer of cancer drug resistance traits by extracellular vesicles. Trends Mol Med. 2015;21(10):595-608.

11. Colombo M, Raposo G, Théry C. Biogenesis, secretion, and intercellular interactions of exosomes and other extracellular vesicles. Annu Rev Cell Dev Biol. 2014;30(1):255-289.

12. Bandari SK, Purushothaman A, Ramani VC, Brinkley GJ, Chandrashekar DS, Varambally S, et al. Chemotherapy induces secretion of exosomes loaded with heparanase that degrades extracellular matrix and impacts tumor and host cell behavior. Matrix Biol. 2018; 65:104-118.

13. Ning $K$, Wang T, Sun X, Zhang P, Chen Y, Jin J, et al. UCH-L1-containing exosomes mediate chemotherapeutic resistance transfer in breast cancer. J Surg Oncol. 2017;115(8):932-940.

14. Kreger BT, Johansen ER, Cerione RA, Antonyak MA. The enrichment of survivin in exosomes from breast cancer cells treated with cisplatin promotes cell survival and chemoresistance. Cancers. 2016;8(12):111. 
15. Livak KJ, Schmittgen TD. Analysis of relative gene expression data using real-time quantitative PCR and the 2(-Delta Delta C(T)) Method. Methods. 2001;25(4):402-408.

16. Lavilla I, Costas M, Miguel PS, Millos J, Bendicho C. Elemental fingerprinting of tumorous and adjacent non-tumorous tissues from patients with colorectal cancer using ICP-MS, ICP-OES and chemometric analysis. Biometals. 2009;22(6):863-875.

17. Vergara $D$, Tinelli A, lannone A, Maffia $M$. The impact of proteomics in the understanding of the molecular basis of Cisplatin-resistance in ovarian tumors. Curr Cancer Drug Targets. 2012;12(8):987-997.

18. Kavallaris M. Microtubules and resistance to tubulin-binding agents. Nat Rev Cancer. 2010;10(3):194-204.

19. Soldevilla B, Rodríguez M, San MC, García V, Fernández PR, Gil CB, et al. Tumor-derived exosomes are enriched in $\triangle N p 73$, which promotes oncogenic potential in acceptor cells and correlates with patient survival. Hum Mol Genet. 2014;23(2):467-478.

20. Zeng AL, Yan W, Liu YW, Wang Z, Hu Q, Nie E, et al. Tumour exosomes from cells harbouring PTPRZ1-MET fusion contribute to a malignant phenotype and temozolomide chemoresistance in glioblastoma. Oncogene. 2017;36(38):5369-5381.

21. Liu X, Fu Q, Du Y, Yang Y, Cho WC. MicroRNA as regulators of cancer stem cells and chemoresistance in colorectal cancer. Curr Cancer Drug Targets. 2016;16(9):738-754.

22. Cui SY, Wang R, Chen LB. MicroRNAs: Key players of taxane resistance and their therapeutic potential in human cancers. J Cell Mol Med. 2013;17(10):1207-1217.

23. Wei F, Ma C, Zhou T, Dong X, Luo Q, Geng L, et al. Exosomes derived from gemcitabine-resistant cells transfer malignant phenotypic traits via delivery of miRNA-222-3p. Mol Cancer. 2017;16(1):132.

24. Qin X, Yu S, Zhou L, Shi M, Hu Y, Xu X, et al. Cisplatin-resistant lung cancer cell-derived exosomes increase cisplatin resistance of recipient cells in exosomal miR-100-5p-dependent manner. Int $\mathrm{J}$ Nanomedicine. 2017; 12:3721-3733.

25. Qu L, Ding J, Chen C, et al. Exosome-Transmitted IncARSR Promotes Sunitinib Resistance in Renal Cancer by Acting as a Competing Endogenous RNA. Cancer Cell. 2016;29(5):653-668.

26. Xu CG, Yang MF, Ren YQ, Wu CH, Wang LQ. Exosomes mediated transfer of IncRNA UCA1 results in increased tamoxifen resistance in breast cancer cells. Eur Rev Med Pharmacol Sci. 2016;20(20):4362-4368.

27. Ciravolo V, Huber V, Ghedini GC, Venturelli E, Bianchi F, Campiglio M, et al. Potential role of HER2overexpressing exosomes in countering trastuzumab-based therapy. J Cell Physiol. 2012;227(2):658667.

28. Schafer T, Maco B, Petfalski E, Tollervey D, Bottcher B, Aebi U, et al. Hrr25-dependent phosphorylation state regulates organization of the pre-40S subunit. Nature. 2006;441(7093):651-655.

29. Kim HD, Kim TS, Kim J. Aberrant ribosome biogenesis activates c-Myc and ASK1 pathways resulting in p53-dependent G1 arrest. Oncogene. 2011;30(30):3317-3327. 
30. Wan F, Anderson DE, Barnitz RA, Snow A, Bidere N, Zheng L, et al. Ribosomal protein S3: a KH domain subunit in NF-kappaB complexes that mediates selective gene regulation. Cell. 2007;131(5):927-939.

31. Kim Y, Kim HD, Kim J. Cytoplasmic ribosomal protein S3 (rpS3) plays a pivotal role in mitochondrial DNA damage surveillance. Biochim Biophys Acta. 2013;1833(12):2943-2952.

32. Kim Y, Yoon JW, Xiao X, Dean NM, Monia BP, Marcusson EG. Selective down-regulation of gliomaassociated oncogene 2 inhibits the proliferation of hepatocellular carcinoma cells. Cancer Res. 2007;67(8):3583-3593.

33. Kool M, Koster J, Bunt J, Hasselt NE, Lakeman A, Sluis PV, et al. Integrated genomics identifies five medulloblastoma subtypes with distinct genetic profiles, pathway signatures and clinicopathological features. PLoS One. 2008;3(8):e3088.

34. Huang Z, Zhang Y, Li H, et al. Vitamin D promotes the cisplatin sensitivity of oral squamous cell carcinoma by inhibiting LCN2-modulated NF-KB pathway activation through RPS3. Cell Death Dis. 2019;10(12):936.

35. Zhao L, Cao J, Hu K, Wang PH, Li GD, He XD, et al. RNA-binding protein RPS3 contributes to hepatocarcinogenesis by post-transcriptionally up-regulating SIRT1. Nucleic Acids Res. 2019;47(4):2011-2028.

36. Tang QF, Sun J, Yu H, Shi XJ, LV R, Wei HC, et al. The Zuo Jin Wan Formula Induces Mitochondrial Apoptosis of Cisplatin-Resistant Gastric Cancer Cells via Cofilin-1. Evidence-Based Complementary and Alternative Medicine. 2016; 2016:1-14.

37. Li M, Yin J, Mao N, Pan L. Upregulation of phosphorylated cofilin 1 correlates with taxol resistance in human ovarian cancer in vitro and in vivo. Oncology Reports. 2013;29(1):58-66.

38. Zhang $\mathrm{H}$, Wang $\mathrm{Y}$, Xing $\mathrm{F}$, et al. Overexpression of LIMK1 promotes migration ability ofmultidrugresistant osteosarcoma cells. Oncology Research. 2012;19(10-11):501-509.

\section{Figures}


A
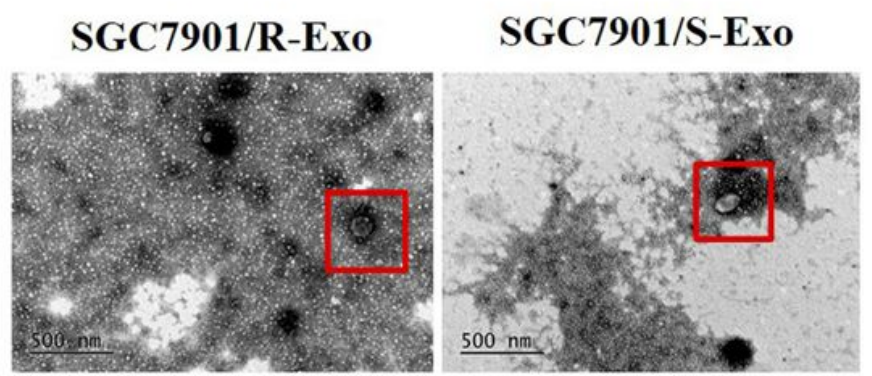

C

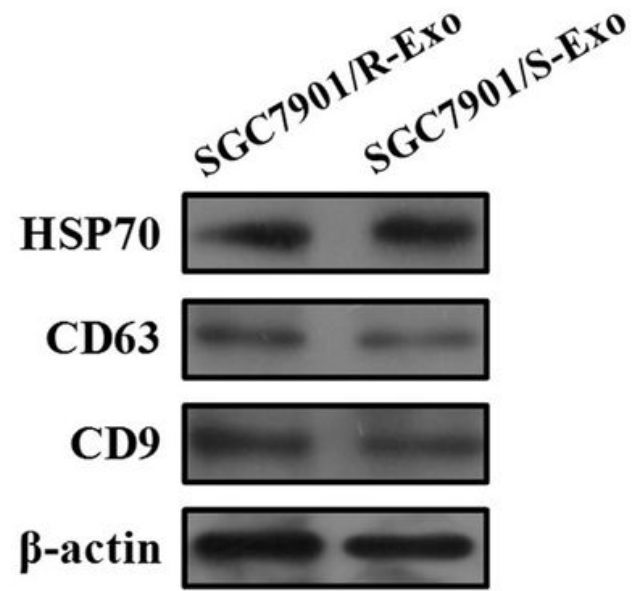

D

DAPI

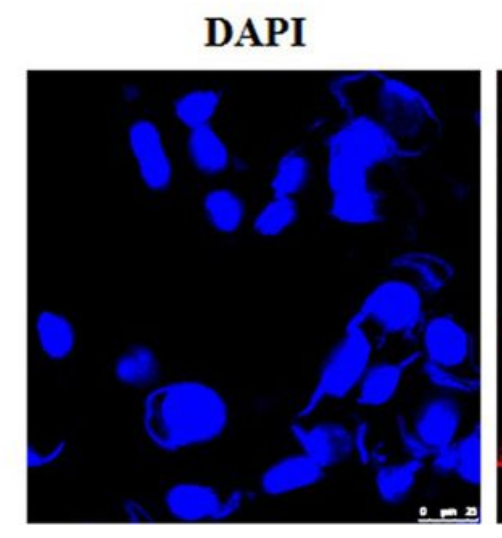

B
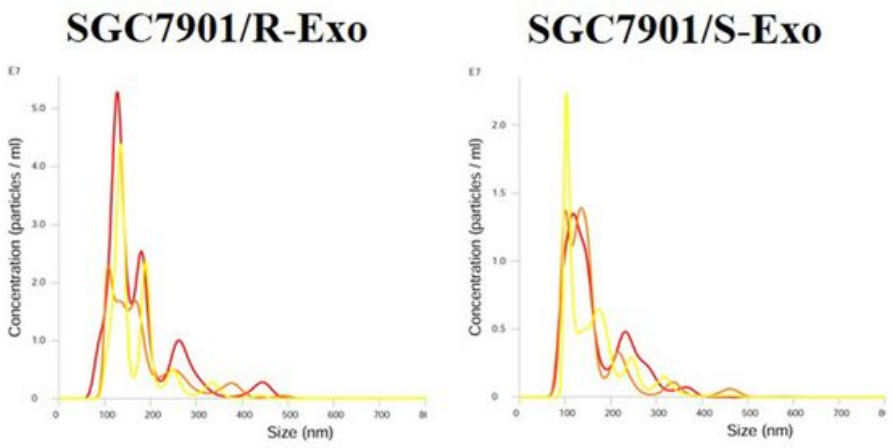

$\mathbf{E}$

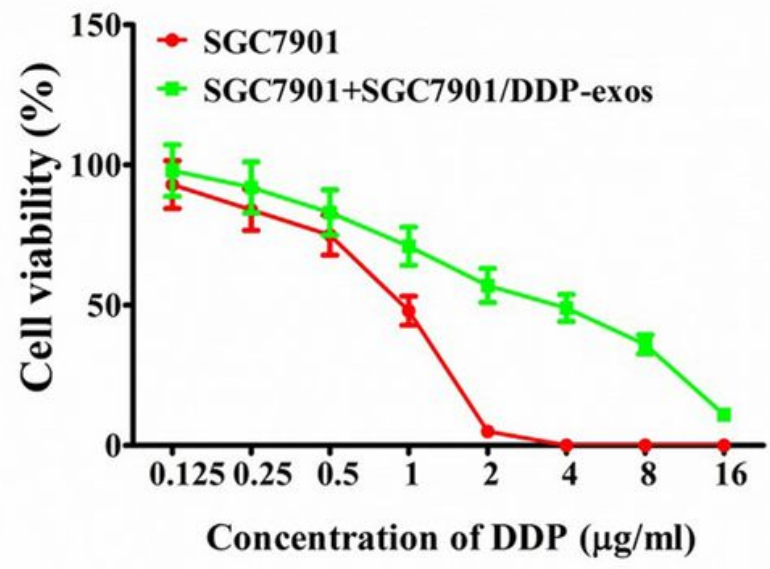

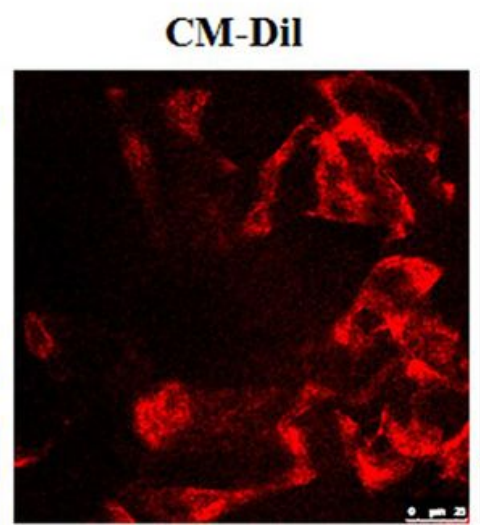
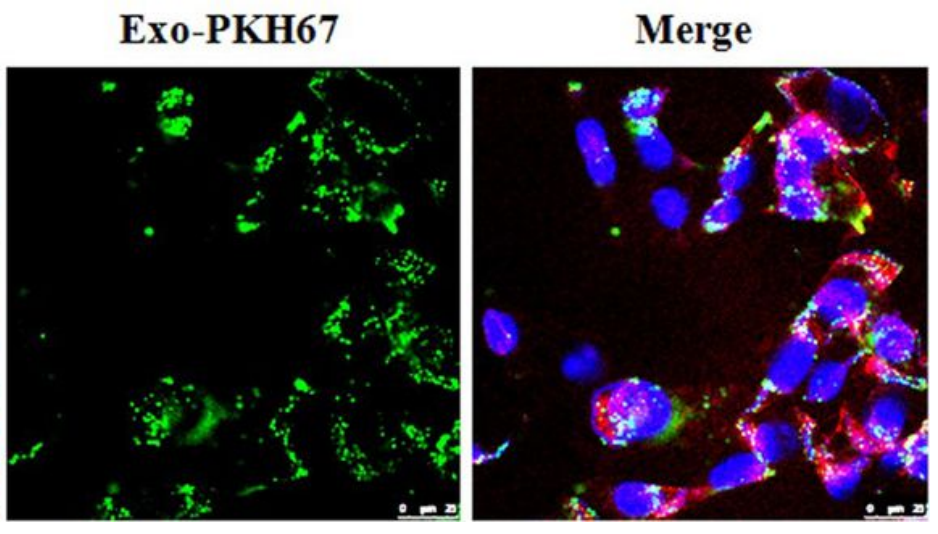

Figure 1

Effect of exosomes derived from SGC7901R cells on the proliferation of SGC7901S cells. (A) Exosome isolation from the cell culture medium of SGC7901R and SGC7901S cells was analyzed for the phenotype (purity and shape) by transmission electron microscopy. The red frames indicate the representative exosomes. Scale bar, $500 \mathrm{~nm}$. (B) SGC7901R and SGC7901S cells were analyzed for size and particle number by the LM10 nanoparticle characterization system. Representative images were provided. (C) Western blotting was performed to detect the exosome markers, including HSP70, CD63, and CD9. $\beta$-actin was used as an internal control. (D) Fluorescence imaging showed the delivery of PKH67-labeled exosomes (green) to CM-Dil-labeled SGC7901S cells (red). Cellular nuclei were stained 
with DAPI. Exosomes were derived from SGC7901R cells. Yellow arrows represented delivered exosomes in the representative images. Scale bar, $25 \mu \mathrm{m}$. (E) CCK-8 assay was performed to observe the effect of cisplatin (DDP) on the proliferation of indicated cells (SGC7901S cells or SGC7901S cells pre-treated with exosomes derived from SGC7901R cells). The concentrations of cisplatin (DDP) used for the drug dose response curve analysis of indicated cells was $0,125,250,500,1000,2000,4000,8000$, and $16000 \mu \mathrm{g} / \mathrm{L}$. Each experiment was performed in triplicate. All the data are shown as mean \pm SD.

A

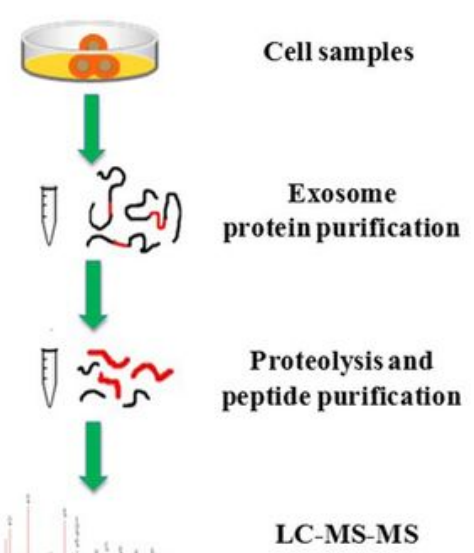

C

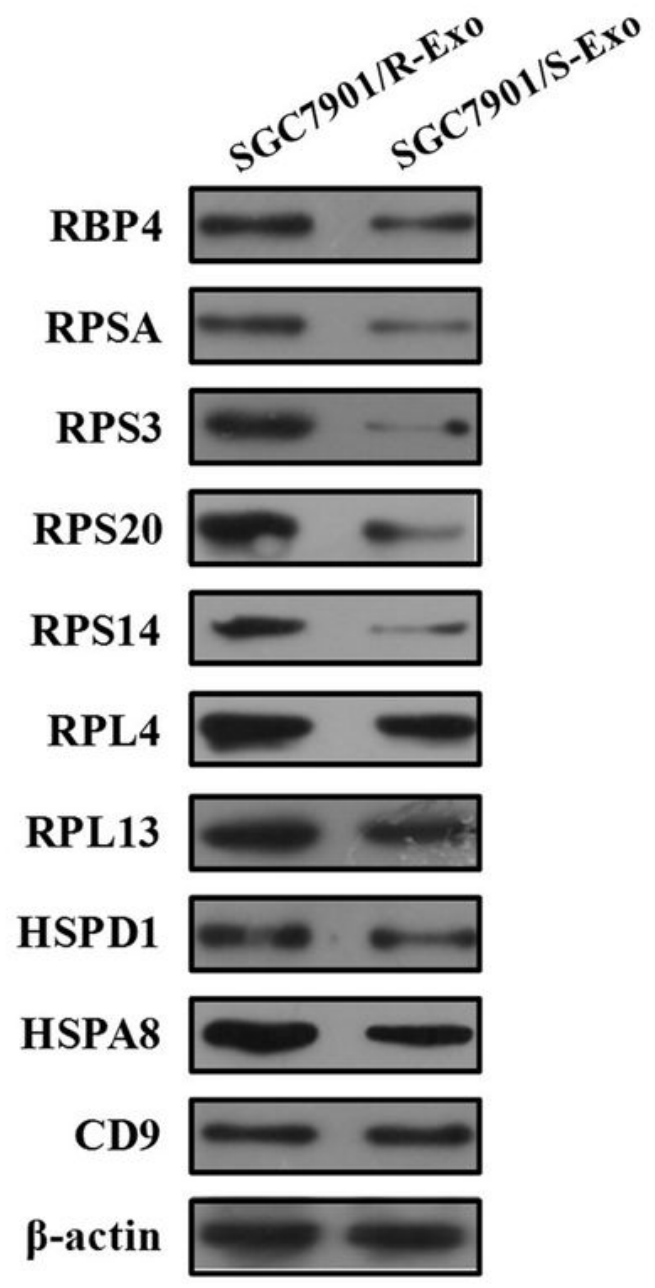

B

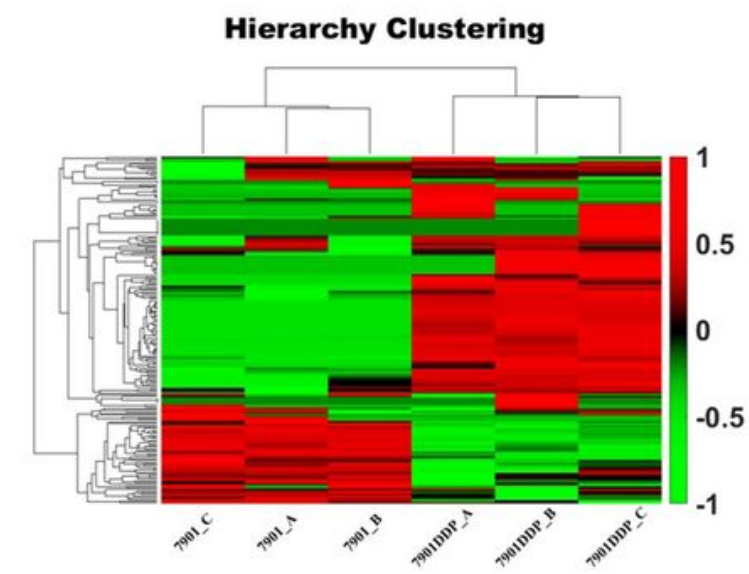

D

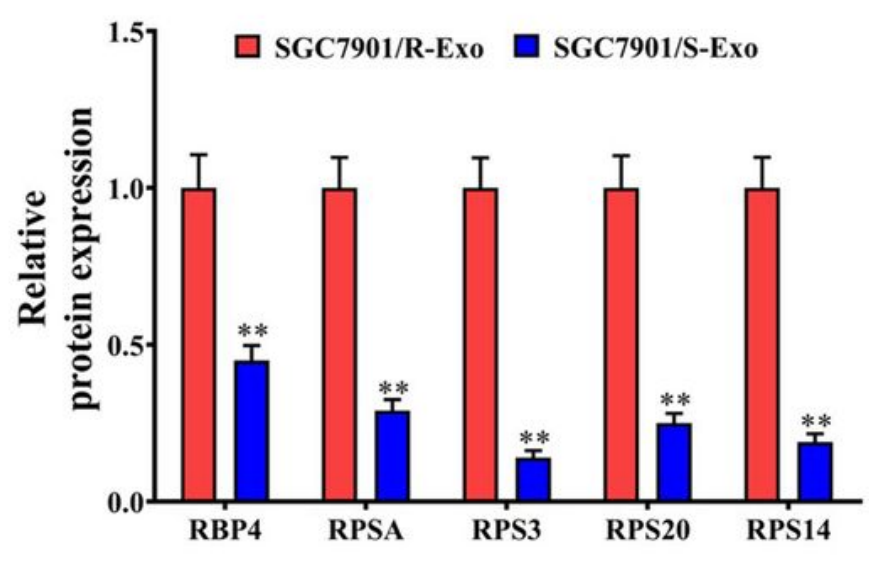

E

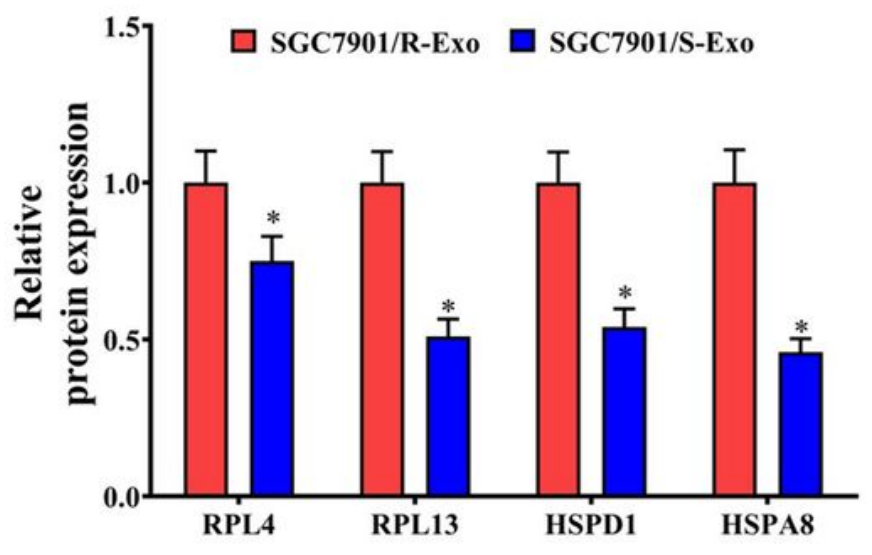

Figure 2 
RPS3 was an important differentially expressed protein between SGC7901R cells exosomes and SGC7901S cells exosomes. (A) The schematic procedure of searching the differentially expressed proteins between SGC7901R cells exosomes and SGC7901S cells exosomes by LC-MS/MS analysis methods. (B) LC-MS/MS and cluster analysis of the differentially expressed proteins between SGC7901R cells exosomes and SGC7901S cells exosomes. Red represents high scores and the green represents low scores. The color brightness of each unit is associated with differences in multiples (log 2[AR/N]). (C, D, E) Western blotting was performed to validate the differentially expressed proteins in the exosomes of SGC7901R and SGC7901S cells. Each experiment was performed in triplicate. All the data are shown as mean \pm SD. Student's t-test was used to analyze the data. **, P $<0.01$ vs SGC7901/R-Exo. 
A

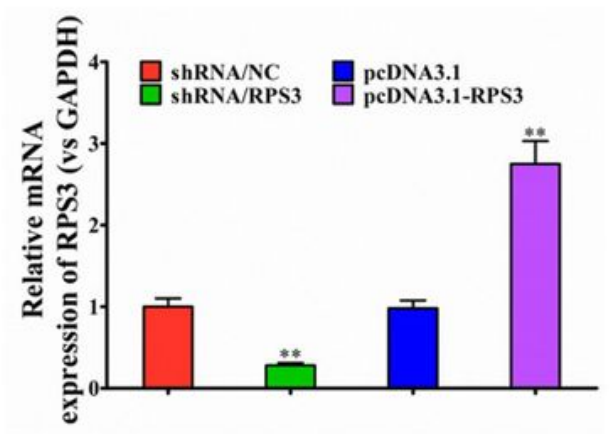

C

RPS3

$\beta$-actin

CD9

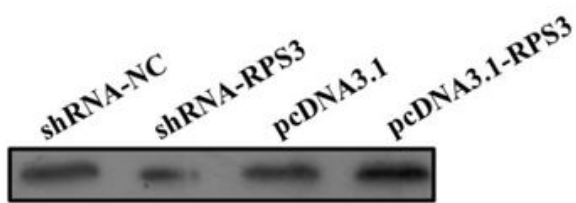

E

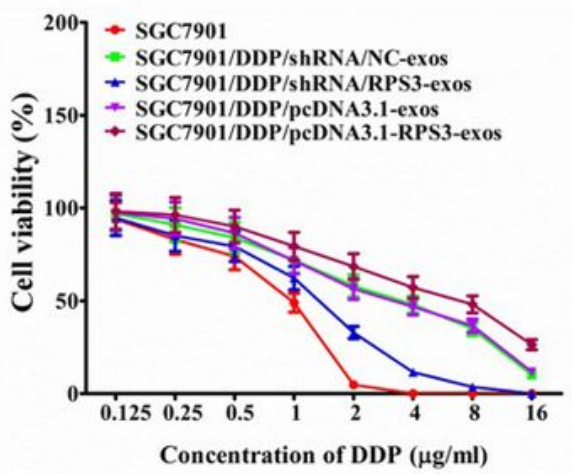

G

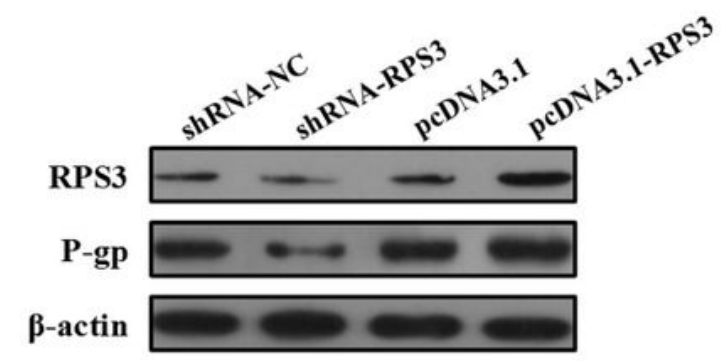

B

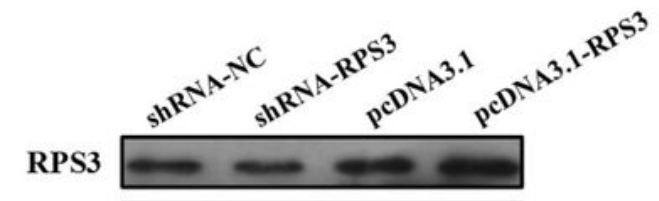

D

$\beta$-actin
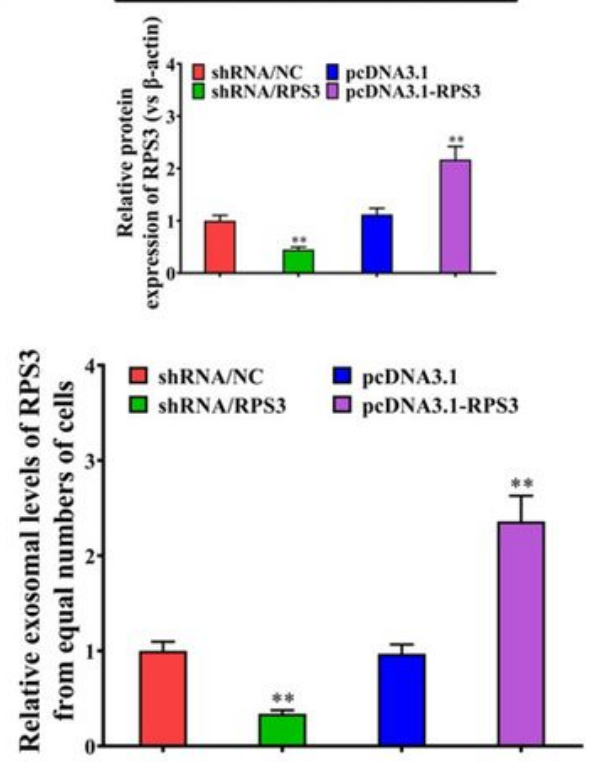

F

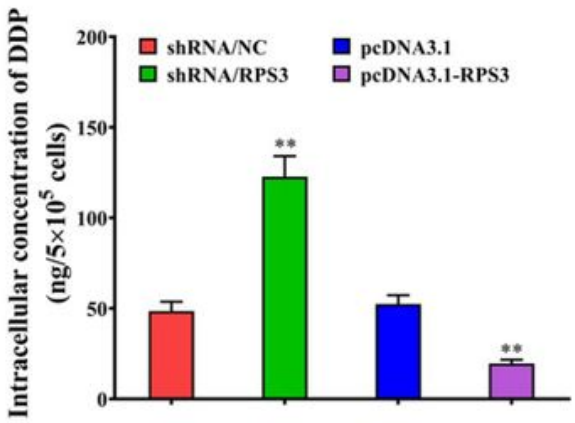

H

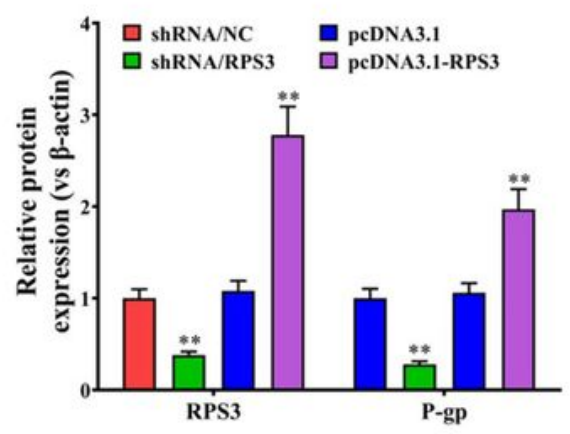

\section{Figure 3}

RPS3 overexpression confers DDP-resistant phenotypes from SGC7901R cells to SGC7901S cells. (A) RT-qPCR was performed to observe the effect of RPS3 knockdown and overexpression on the mRNA expression of RPS3 in SGC7901R cells. (B) Western blotting was performed to observe the effect of RPS3 knockdown and overexpression on the protein expression of RPS3 in SGC7901R cells. (C, D) Western blotting and quantitative analysis were performed to observe the effect of RPS3 knockdown and 
overexpression on the exosomal expression of RPS3 protein in SGC7901R cells. $\beta$-actin was used as cellular control, and CD9 was set as the marker of exosomes. (E) CCK-8 assay was performed to observe the effect of cisplatin (DDP) on the proliferation of indicated cells: SGC7901S, SGC7901S+ SGC7901RshRNA/NC-exos, SGC7901S+ SGC7901R-shRNA/RPS3-exos, SGC7901S+ SGC7901R-pcDNA3.1-exos, SGC7901S+ SGC7901R-pcDNA3.1-RPS3-exos. The concentrations of DDP used for the drug dose response curve analysis of indicated cells were $0,125,250,500,1000,2000,4000,8000$, and $16000 \mu \mathrm{g} / \mathrm{L}$. Each experiment was performed in triplicate. (F) ICP-MS analysis was applied to see the effect of exosomes from indicated cells (SGC7901SR-shRNA/NC, SGC7901SR-shRNA/RPS3, SGC7901SRpcDNA3.1, SGC7901SR-pcDNA3.1-RPS3) on the DDP concentration in the cellular of SGC7901S cells. (G, $\mathrm{H})$ Western blotting and quantitative analysis were performed to observe the effect of exosomes from indicated cells (SGC7901SR-shRNA/NC, SGC7901SR-shRNA/RPS3, SGC7901SR-pcDNA3.1, SGC7901SRpcDNA3.1-RPS3) on the P-gp protein expression in SGC7901S cells. $\beta$-actin was used as cellular control. All the data are shown as mean \pm SD. Student's t-test was used to analyze the data. ${ }^{\star \star}, P<0.01$ vs SGC7901R-shRNA/NC. 
A

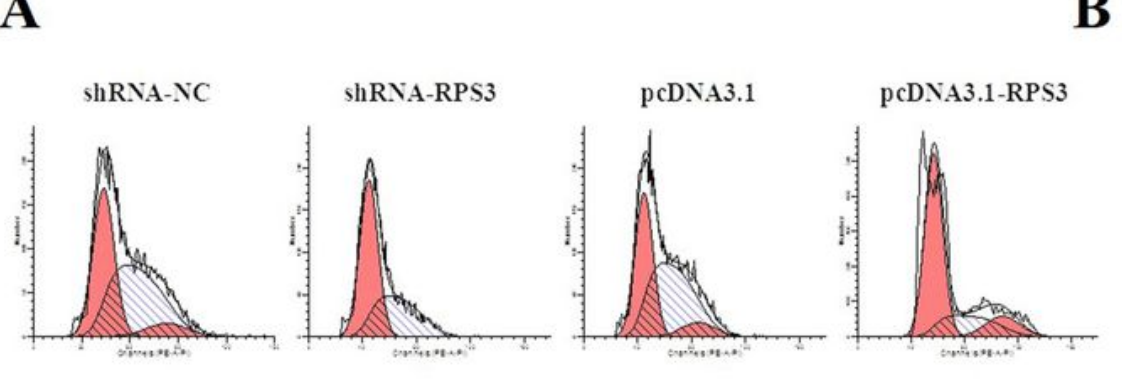

C

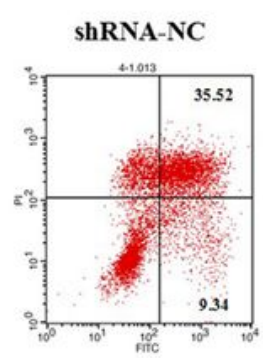

$\mathbf{E}$

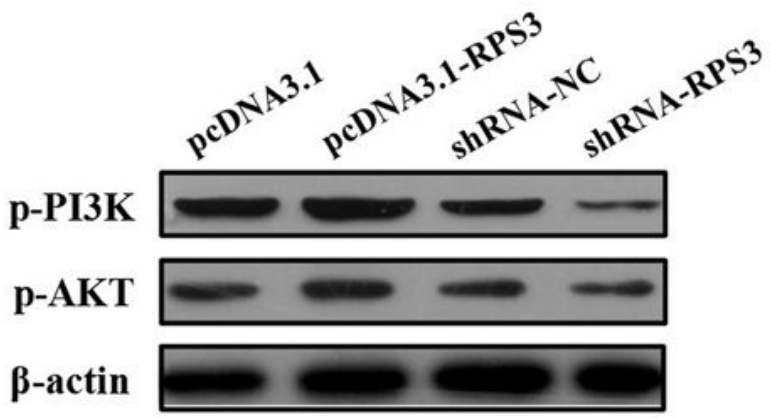

G

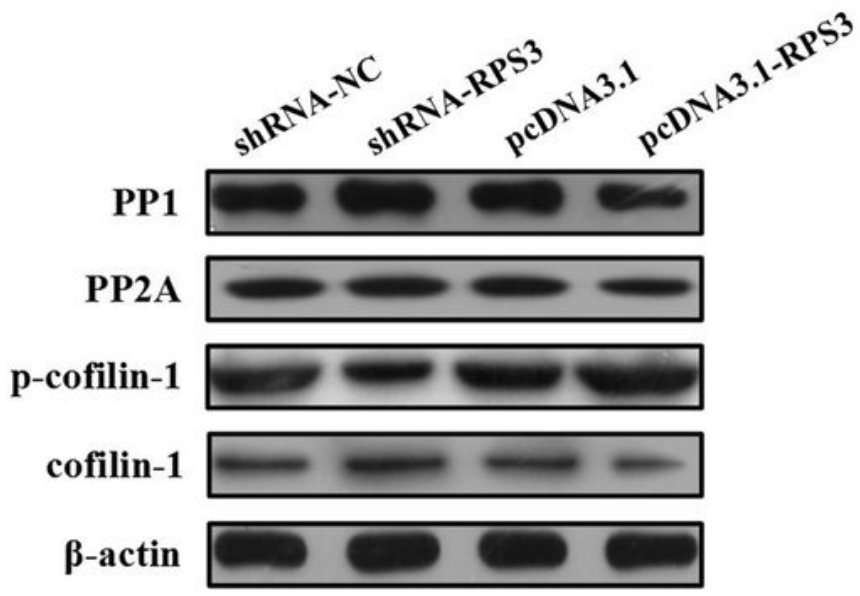

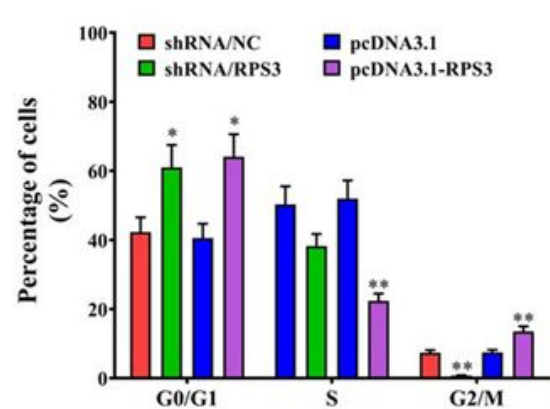

D

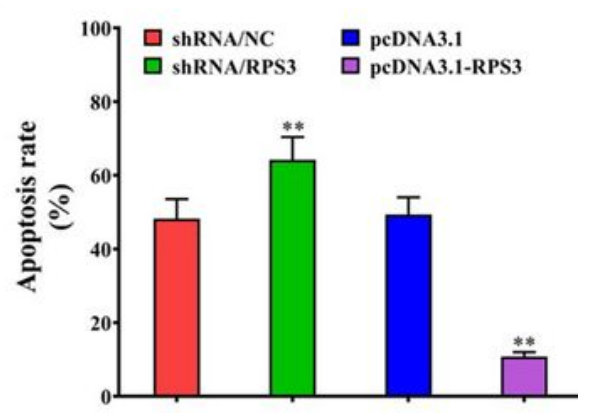

$\mathbf{F}$

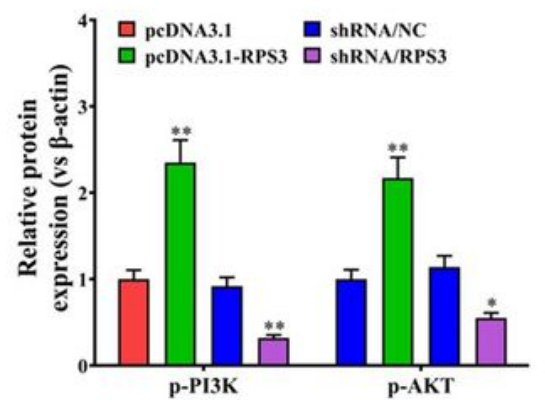

H

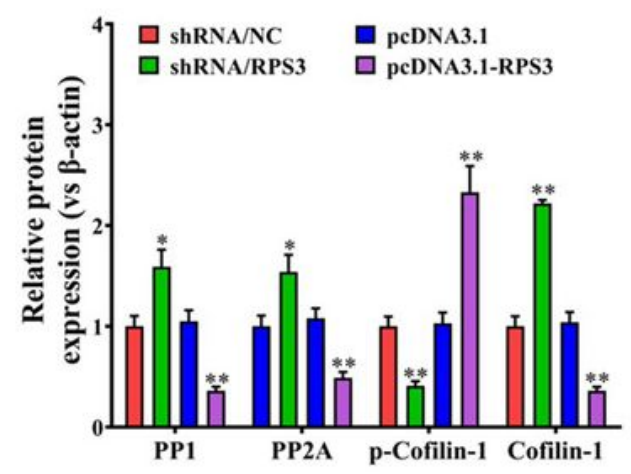

\section{Figure 4}

RPS3-riched exosomes from SGC7901R inhibited the apoptosis of SGC7901S via mitochondrial translocation of p-cofilin-1. (A, B) Flow cytometry method and quantitative assay were performed to observe the shift of cell cycles in SGC7901S cells treated with exosomes derived from RPS3overexpressing or -silencing SGC7901R cells, or their respective controls. (C, D) Flow cytometry method and quantitative assay were performed to observe the change of cell apoptosis in SGC7901S cells treated 
with exosomes derived from RPS3-overexpressing or -silencing SGC7901R cells, or their respective controls. (E-H) Western blotting and quantitative assays of p-PI3K, p-AKT, PP1, PP2A, p-cofilin-1, and cofilin-1 proteins in the SGC7901S cells treated with exosomes derived from RPS3-overexpressing or silencing SGC7901R cells, or their respective controls. Each experiment was performed at least in triplicate and all the data are shown as mean \pm SD. Student's t-test was used to analyze the data. ${ }^{*} \mathrm{P}<$ $0.05 ; * *, P<0.01$.

A

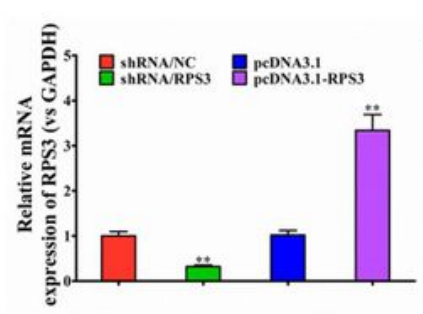

D

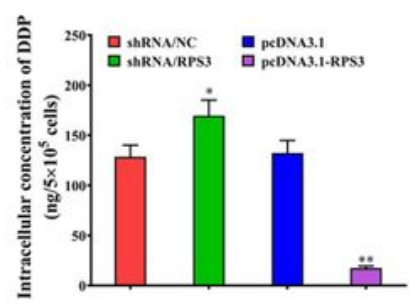

G
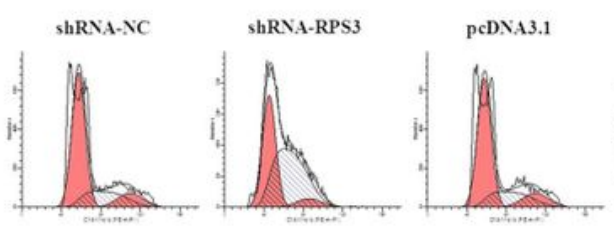

$\mathbf{E}$
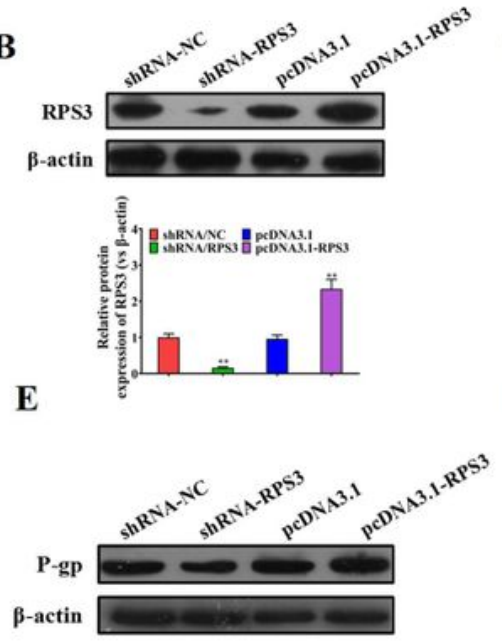

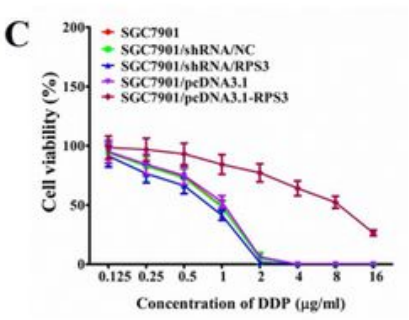

F

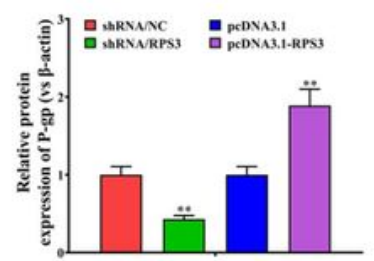

H
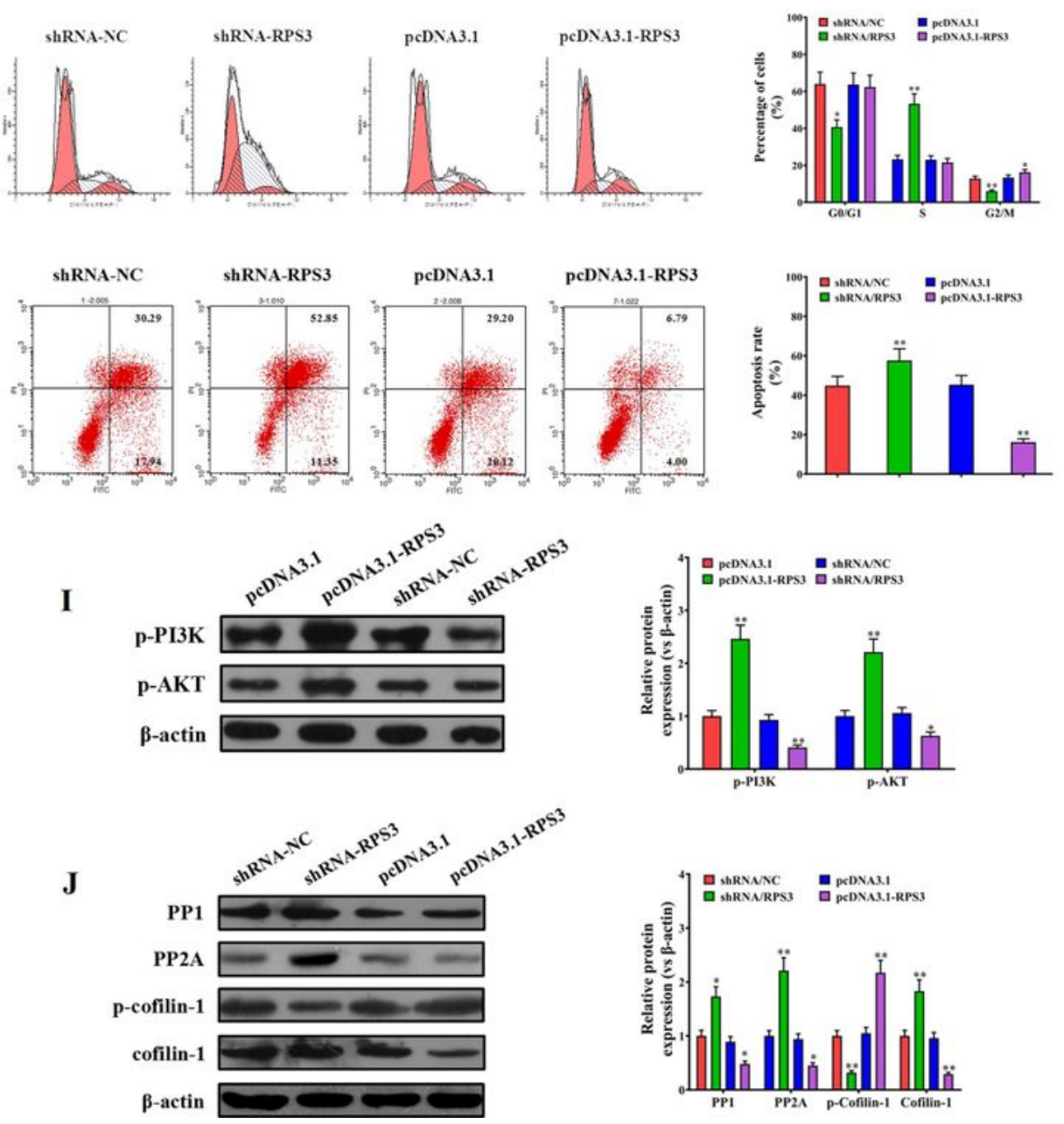

Figure 5

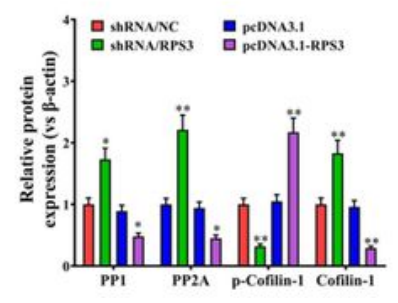


RPS3 overexpression directly in SGC7901S cells increases the DDP-resistance of SGC7901S cells. (A) RTqPCR was performed to observe the effect of RPS3 knockdown and overexpression on the mRNA expression of RPS3 in SGC7901S cells. (B) Western blotting was performed to observe the effect of RPS3 knockdown and overexpression on the protein expression of RPS3 in SGC7901S cells. (C) CCK-8 assay was performed to observe the effect of cisplatin (DDP) on the proliferation of indicated cells: SGC7901S, SGC7901S-shRNA/NC, SGC7901S+ SGC7901S-shRNA/RPS3, SGC7901S-pcDNA3.1, SGC7901SpcDNA3.1-RPS3. The concentrations of DDP used for the drug dose response curve analysis of indicated cells were $0,125,250,500,1000,2000,4000,8000$, and $16000 \mu \mathrm{g} / \mathrm{L}$. Each experiment was performed in triplicate. (D) ICP-MS analysis was applied to investigate the effect of RPS3 knockdown and overexpression on the DDP concentration in the cellular of SGC7901S cells. (E, F) Western blotting and quantitative analysis were performed to observe the effect of RPS3 knockdown and overexpression on the P-gp protein expression in SGC7901S cells. $\beta$-actin was used as cellular control. (G) Flow cytometry and quantitative assay were performed to observe the shift of cell cycles in SGC7901S cells treated with RPS3-overexpressing or -silencing, or their respective controls. $(\mathrm{H})$ Flow cytometry and quantitative assay were performed to observe the change of cell apoptosis in SGC7901S cells treated with RPS3overexpressing or -silencing, or their respective controls. (I-J) Western blotting and quantitative assays of p-PI3K, p-AKT, PP1, PP2A, p-cofilin-1, and cofilin-1 proteins in the SGC7901S cells treated with RPS3overexpressing or -silencing, or their respective controls. Each experiment was performed at least in triplicate and all the data are shown as mean \pm SD. Student's t-test was used to analyze the data. ${ }^{*}, \mathrm{P}<$ $0.05 ; * \star, P<0.01$. 
A

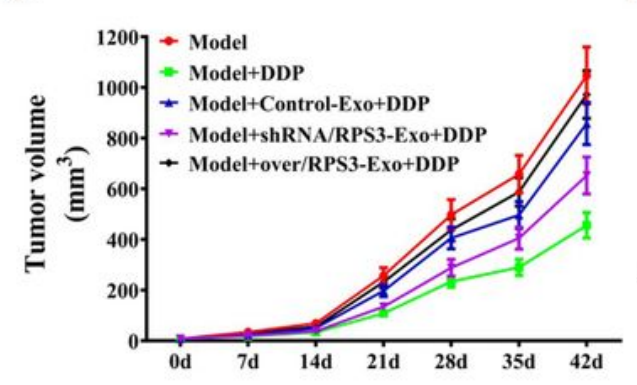

B

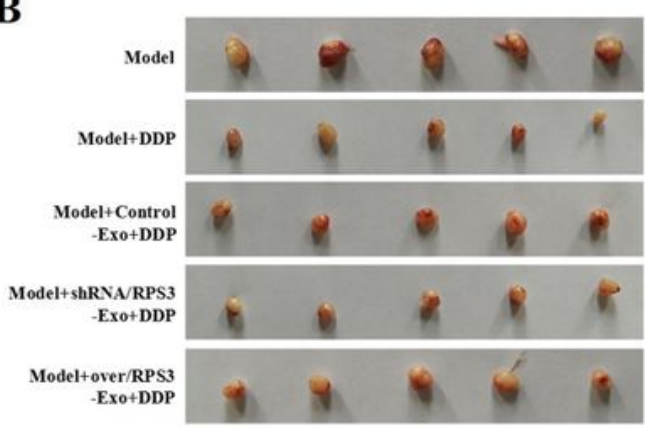

C

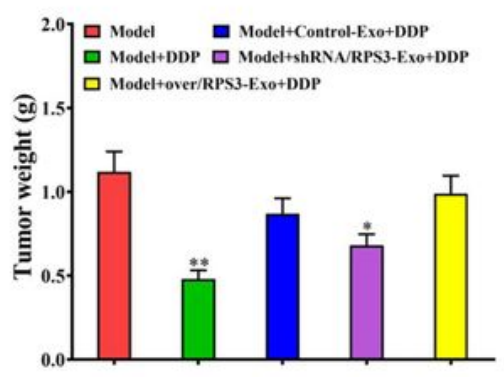

D

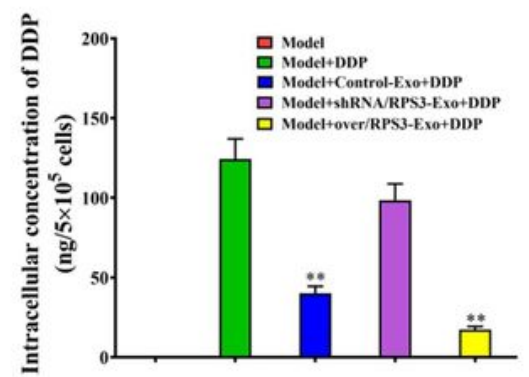

$\mathbf{E}$

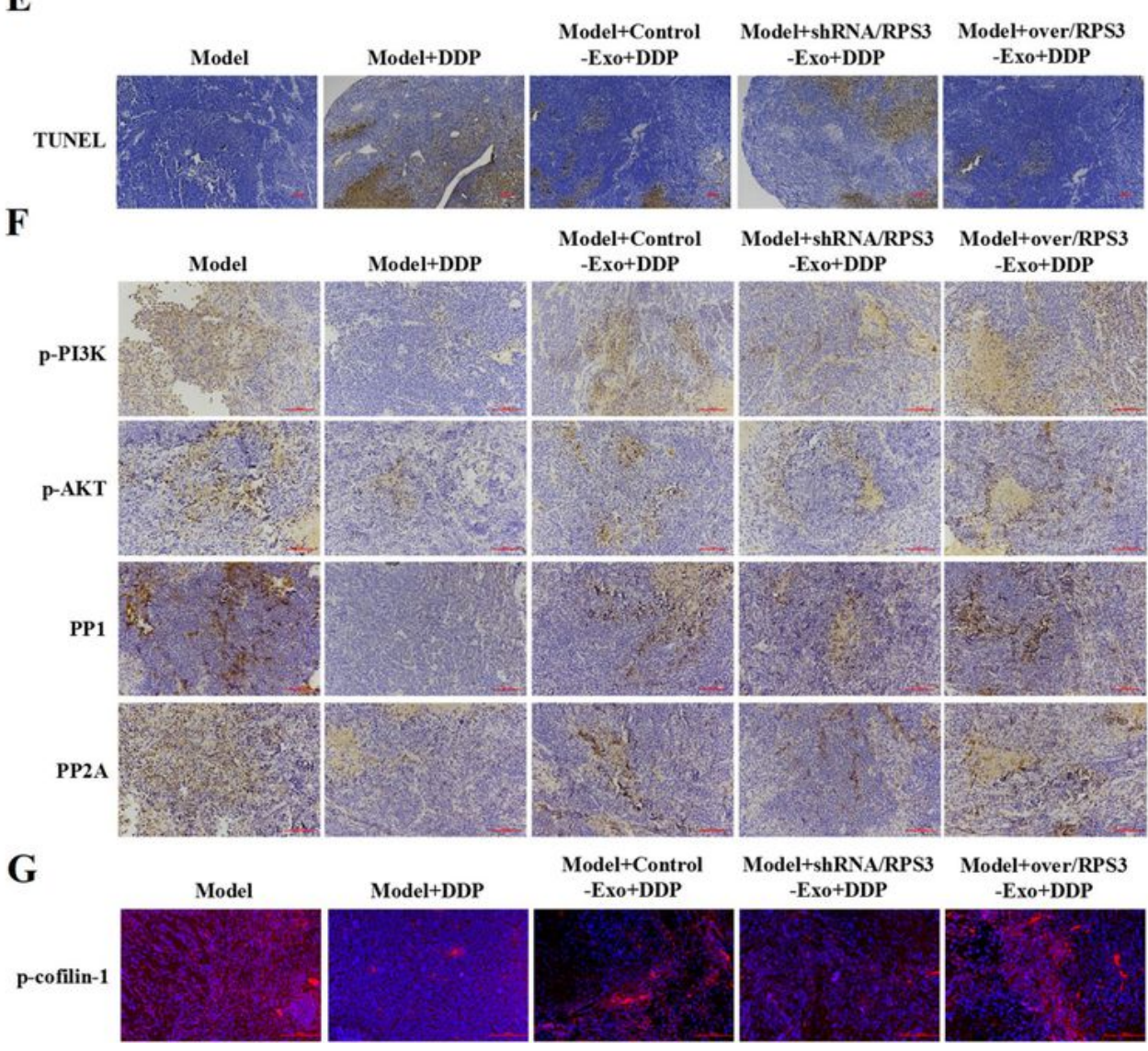

\section{Figure 6}

Impact of RPS3-riched exosomes from SGC7901R on the growth of subcutaneous xenograft of SGC7901S. (A) Xenografts of SGC-7901S cells with indicated treatments performed on nude mice. SGC$7901 \mathrm{~S}$ cells were pretreated with exosomes from RPS3-overexpressing or -silencing SGC-7901R cells, or control SGC-7901R cells. The length and width of the subcutaneous tumors were measured every 7 days. $(B, C)$ Tumors were surgically removed from nude mice and weighed after administration for 35 days. *, $P$ 
$<0.05 ; * \star, P<0.01$, in contrast to model groups treated with DDP alone. (D) ICP-MS analysis was applied to investigate the DDP concentration in the subcutaneous xenograft of SGC7901S cells with indicated treatments. (E) TUNEL assay was performed to observe the apoptosis of tumor cells in the subcutaneous xenograft of SGC7901S cells with indicated treatments. Scale bar, $100 \mu \mathrm{m}$. (F) The xenograft tumor tissues in mice of all groups were subjected to immunohistochemistry analysis using the antibody of $p$ PI3K, p-AKT, PP1, and PP2A. Scale bar, $100 \mu \mathrm{m}$. (G) Immunofluorescence detection of the expression of pcofilin-1 in the subcutaneous xenograft of SGC7901S cells with indicated treatments. Scale bar, $100 \mu \mathrm{m}$. Each experiment was performed at least in triplicate and all the data are shown as mean \pm SD. Student's t-test was used to analyze the data. *, $\mathrm{P}<0.05 ; * \star, \mathrm{P}<0.01$.

\section{Supplementary Files}

This is a list of supplementary files associated with this preprint. Click to download.

- FigureS1.jpg

- FigureS1.jpg

- FigureS2.jpg

- Figures2.jpg

- FigureS3.jpg

- FigureS3.jpg

- SupplementaryMaterial.docx

- SupplementaryMaterial.docx 